\title{
Analysis of Acoustic Communication Channel Characterization Data in the Surf Zone
}

\author{
by \\ James Willard Partan \\ B.A., Williams College, 1994 \\ Submitted to the Department of Electrical Engineering and Computer Sciencé \\ in partial fulfillment of the requirements for the degree of \\ Master of Science in Electrical Engineering \\ at the \\ MASSACHUSETTS INSTITUTE OF TECHNOLOGY \\ and the \\ WOODS HOLE OCEANOGRAPHIC INSTITUTION
}

September 2000

(C)James W. Partan 2000. All rights reserved.

The author hereby grants to MIT and WHOI permission to reproduce paper and electronic copies of this thesis in whole or in part and to distribute them publicly.

Author

Department of Electrical Engineering and Computer Science, Massachusetts Institute of Technology, and the Joint Program in Oceanography and Oceanographic Engineering, Massachusetts Institute of Technology/Woods Hole Oceanographic Institution August 4, 2000

Certified by

James C. Preisig

Assistant Scientist, Woods Hole Oceanographic Institution Thesis Supervisor

Accepted by .

Michael S. Triantafyllou

Chair, MYT/WHOJ Jonét Committee on Oceanographic Engineering

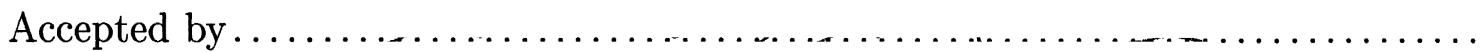
Arthur Q. Smith Chair, MIT Electrical Engineering Department Committee on Graduate Students 


\title{
Analysis of Acoustic Communication Channel Characterization Data in
}

\section{the Surf Zone}

\author{
by
}

\author{
James Willard Partan
}

\author{
Submitted to the Department of Electrical Engineering and Computer Science \\ on August 4, 2000, in partial fulfillment of the \\ requirements for the degree of \\ Master of Science in Electrical Engineering
}

\begin{abstract}
A channel characterization experiment for the underwater acoustic communication channel was carried out at Scripps Pier in May 1999. The experiment investigated acoustic transmission in very shallow water and breaking waves. In analyzing the data, several questions arose.

The majority of the acoustic channel probe data was corrupted by crosstalk in the receiver array cable. This thesis investigates methods to correct for the effects of the crosstalk, to attempt to recover the channel probe data. In selected regions, the crosstalk could be removed quite effectively using a linear least-squares method to estimate the crosstalk coefficients. The bulk of the data could not be corrected, however, primarily due to crosstalk from a receiver channel which was not recorded, and hence could not be well estimated.
\end{abstract}

A second question addressed by this thesis is concerned with acoustic propagation in shallow water under bubble clouds. The breaking waves injected air deep into the water column. The resulting bubble clouds heavily attenuated acoustic signals, effectively causing total dropouts of the acoustic communication channel. Due to buoyancy, the bubbles gradually rise, and the communication channel clears. The channel clearing was significantly slower than predicted by geometric ray acoustic propagation models, however. Proposed explanations included secondary, unobserved, breaking events causing additional bubble injection; delayed rising of bubbles due to turbulent currents; or failure of the geometric ray model due to suppression by bubble clouds of acoustic signals which are not along the geometric ray paths. This thesis investigated the final hypothesis, modeling the acoustic propagation in Scripps Pier environment, using the full wave equation modeling package OASES. It was determined that the attenuation of the propagating acoustic signal is not accurately predicted by the bubble-induced attenuation along the geometric ray path.

Thesis Supervisor: James C. Preisig

Title: Assistant Scientist, Woods Hole Oceanographic Institution 


\section{Acknowledgments}

First, I would like to sincerely thank my advisor, Jim Preisig, for his patience, positive attitude, advice, and expertise. I would especially like to thank Mark Johnson for his extensive help on all matters and for his friendship. Thanks also to my friends and colleagues at the Woods Hole Oceanographic Institution, including Patrick Miller, Nicoletta Biassoni, Jon Woodruff, Doug Nowacek, Lee Freitag, Gene Terray, Matt Grund, Tom Hurst, Alex Shorter, and many others. Most importantly, thanks to my parents and brother for their love and support.

For financial support, thanks to the National Science Foundation for funding me on a Graduate Research Fellowship, and thanks to the WHOI Education Office for supplementing that fellowship. 


\section{Contents}

1 Introduction 5

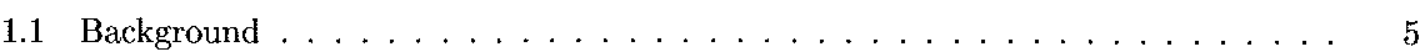

1.2 Experimental Approach $\ldots \ldots \ldots \ldots \ldots \ldots$

1.3 Data Analysis, Crosstalk Correction, and Propagation Modeling . . . . . . . 8

2 Scripps Pier Hydrophone Cable Crosstalk:

Analysis and Correction $\quad 9$

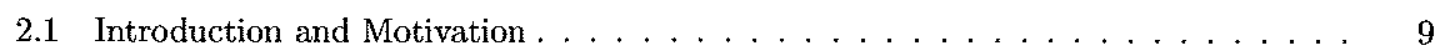

2.2 Crosstalk Model . . . . . . . . . . . . . . . . . . . . . . . . . . 11

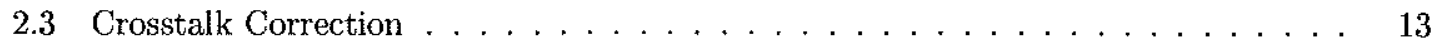

2.4 Results . . . . . . . . . . . . . . . . . . . . . 23

2.5 Alternative approaches . . . . . . . . . . . . . . . . . 29

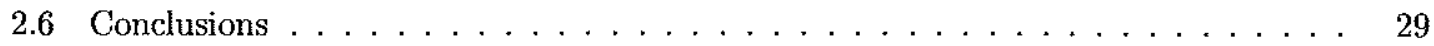

3 Acoustic Propagation Modeling in Bubble Clouds 32

3.1 Introduction and Motivation . . . . . . . . . . . . . . . . . . . . 32

3.2 Modeling Approach . . . . . . . . . . . . . . . . . . . . 33

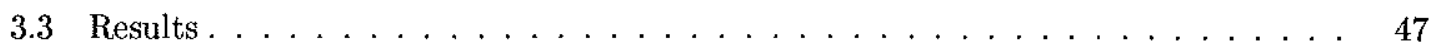

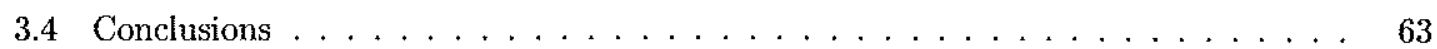

$\begin{array}{ll}\text { Bibliography } & 64\end{array}$ 


\section{Chapter 1}

\section{Introduction}

\subsection{Background}

Very shallow water and the surf zone present a challenging channel for underwater acoustic communication. Characteristics of the channel include rapidly varying multipath structure and associated fading, high levels of ambient noise, and periodic channel dropouts where signal transmission is impossible.

Most of the past work in acoustic underwater communications has been done in relatively deep water, and even the work in shallow water - roughly defined as regions where the transmission range is greater than ten times the water depth - has avoided the adverse conditions of the surf zone $[1,18]$.

Recently, however, there has been interest in extending underwater acoustic communication networks into very shallow water and the surf zone, in particular for mine location and clearing operations conducted by small autonomous underwater vehicles. Underwater communication networks will provide command and navigation data from master nodes to the vehicles, and high-rate data uplink from the vehicles to the nodes. Characterization of the channel in the surf zone would allow the communication systems to be closely matched to the environment, by determining what the best frequency bands are, what modulation methods can be supported, and what coding and protocols will be necessary. In addition, understanding the correlation of the channel parameters with environmental conditions will allow realistic expectations for the performance of the communication system, given a site's particular bathymetry, wind, and sea states[14]. 
Over the past few years, several major scientific experiments have been conducted to study the acoustic transmission properties of the surf zone, but the experiments have concentrated on the physics of air-sea interactions and breaking waves, rather than determining engineering parameters for the underwater acoustic communication channel[20,19]. The primary goal of these acoustic experiments has been to study the bubbles formed in breaking waves, which are the main source of both ambient noise and attenuation in the surf zone. Heavy attenuation can lead to total channel dropouts, which can last for over a minute, with attenuations of up to $26 \mathrm{~dB} /$ meter[6]. The past experiments have mainly examined the higher frequency bands, where most of the bubble resonances lie. From an acoustic communications perspective, however, characterization of the bands with less impact from bubbles, in the range from approximately $4-20 \mathrm{kHz}$, is more important. Lower frequencies are less attractive because low-frequency transducers are too bulky for use on a small vehicle, and because diffraction effects become more pronounced as the acoustic wavelength becomes non-negligible compared with inhomogeneities in the propagation environment. Higher frequencies, while offering a larger bandwidth, are attenuated more severely by the bubbles, and are incompatible with readily-available existing acoustic communication hardware[22].

In addition to bubbles formed in breaking surf, another major problem for communication in the very shallow water channel will be the rapidly varying multipath structure[2]. The slow multipath evolution is driven by the tidal cycle, while the rapid multipath fluctuation is primarily due to scattering from the surface wave field. Fluctuation of the multipath impulse response leads directly to a finite channel coherence time, the length of which will heavily influence the design of modulation schemes and packet sizes. Measurements of the Doppler spreads of individual arrivals within the multipath structure, and of the angle of arrival of the different paths, could help in designing adaptive beamforming array equalizers to select the minimally-Doppler-spread direct path arrival.

\subsection{Experimental Approach}

In May 1999, a large channel characterization experiment was conducted at the Scripps Institution of Oceanography (SIO). Its purpose was to characterize the acoustic telemetry channel in very shallow water and the surf zone. The motivation for this work was to aid in the development of reliable communication methods for the surf zone environment. 
The probe signals were chosen in order to characterize as many parameters as possible of the timevarying communication channel. In particular, the transmission loss and ambient noise, available bandwidth, the multipath structure and its time-delay spreading, and the coherence time, all needed to be measured. An ideal probe signal would be similar to a data packet, i.e. approximating white noise, filling the frequency band of the transmitter, and as long as a data packet. Repeated maximal-length pseudorandom noise sequences (M-sequences) match these requirements well, particularly for phase-shift key (PSK) direct-sequence spread spectrum signals. They have an autocorrelation resembling that of white noise, and provide a high processing gain[16]. In addition, all of the parameters mentioned above can be determined from processed maximal-length sequence transmissions, using standard approaches to channel characterization[8]. The Scripps Pier experiment primarily transmitted M-sequences and pseudorandom sequences with similar properties.

In addition to the transmission of acoustic channel probes, simultaneous and comprehensive measurements of the physical environmental conditions driving the time-varying channel were made. These additional physical measurements included fairly simple measurements, such as tidal cycle, wind speed and direction, and overhead video of the site, as well as more involved spatial and temporal measurements of the underwater bubble cloud locations and bubble size distribution, the current fields advecting the bubble clouds, and array measurements of the wave and temperature fields[5].

The acoustic channel probe signals were transmitted from several different sources, covering the frequency bands from 4-20 kHz. These sources were located on both sides of the heaviest surf, so that during channel dropouts in the surf zone, the hydrophones on either side of the breaking waves would still receive some signal. The hydrophones were deployed in a large horizontal array extending approximately 300 meters across the surf zone. At the ends of the horizontal array, there were two smaller vertical arrays, each roughly spanning the vertical water column.

Each packet of probe signals was preceded by a frequency-shift key (FSK) sequence to act as a synchronization signal for data processing. The FSK synchronization signal has been well-tested, and is robust in multipath channels, whereas a phase-shift key (PSK) modulation technique, as was used for the $\mathrm{M}$-sequence probes, may not be adequate for synchronization due to channel coherence limitations and ambient noise levels. In addition to the synchronization and M-sequence probes, small packets of sample communication packets were transmitted, testing various modulation schemes. The entire packet of signals was repeated up to several times per minute, sharing 
transmission time with a sonar system which was used to map the bubble clouds.

\subsection{Data Analysis, Crosstalk Correction, and Propagation Modeling}

Although the Scripps Pier experiment produced a very valuable data set overall, many of the hydrophone channels were heavily contaminated by crosstalk. Chapter 2 of this thesis investigates methods to estimate the crosstalk coefficients between channels, and to then correct the recorded signals for the crosstalk. Correcting the acoustic probe recordings for the crosstalk would recover a critical piece of the overall environmental measurements.

With breaking waves injecting bubble clouds into the water column, the surf zone often has long channel outages. The communication system's protocol, coding, and modulation must take into account these extended outages. In order to model system performance and test new techniques, realistic models of the surf zone environment must be created. After analysis of the Scripps Pier channel characterization data, it was also determined that there was possibly a discrepancy in the time for the acoustic communication channel to clear between modeled and measured results. The time for the acoustic communication channel to clear after an outage was measured, then modeled with a geometric ray model for acoustic propagation. The discrepancy between these results led to the investigation in Chapter 3 of this thesis, in which a full wave equation propagation model was used to model acoustic propagation in the surf zone. 


\section{Chapter 2}

\section{Scripps Pier Hydrophone Cable}

\section{Crosstalk:}

\section{Analysis and Correction}

\subsection{Introduction and Motivation}

A primary goal of the May 1999 experiment at Scripps Pier was to determine correlations between environmental conditions and the time-varying characteristics of the underwater acoustic communications channel. Therefore, in addition to the three groups characterizing the surf zone communications channel, two additional teams of investigators were simultaneously measuring many environmental variables at high temporal resolutions[21]. The resulting data sets of acoustic channel probes and local physical conditions are very valuable, and would be extremely timeconsuming and expensive to reproduce.

During the course of the Scripps Pier channel characterization experiment, it was noticed that signals were appearing simultaneously on widely-separated hydrophones, without the expected acoustic propagation delay, as shown in Figure 2-1. This problem was later traced to a systematic miswiring of the hydrophone cable, which capacitively coupled the channels together. The energy in these crosstalk signals was roughly $35 \mathrm{~dB}$ lower than the energy in the acoustically-received signals creating them. The transmission loss across the channel, however, was almost always over $40 \mathrm{~dB}$, so the crosstalk signals were in general stronger than the acoustically-received signals on 


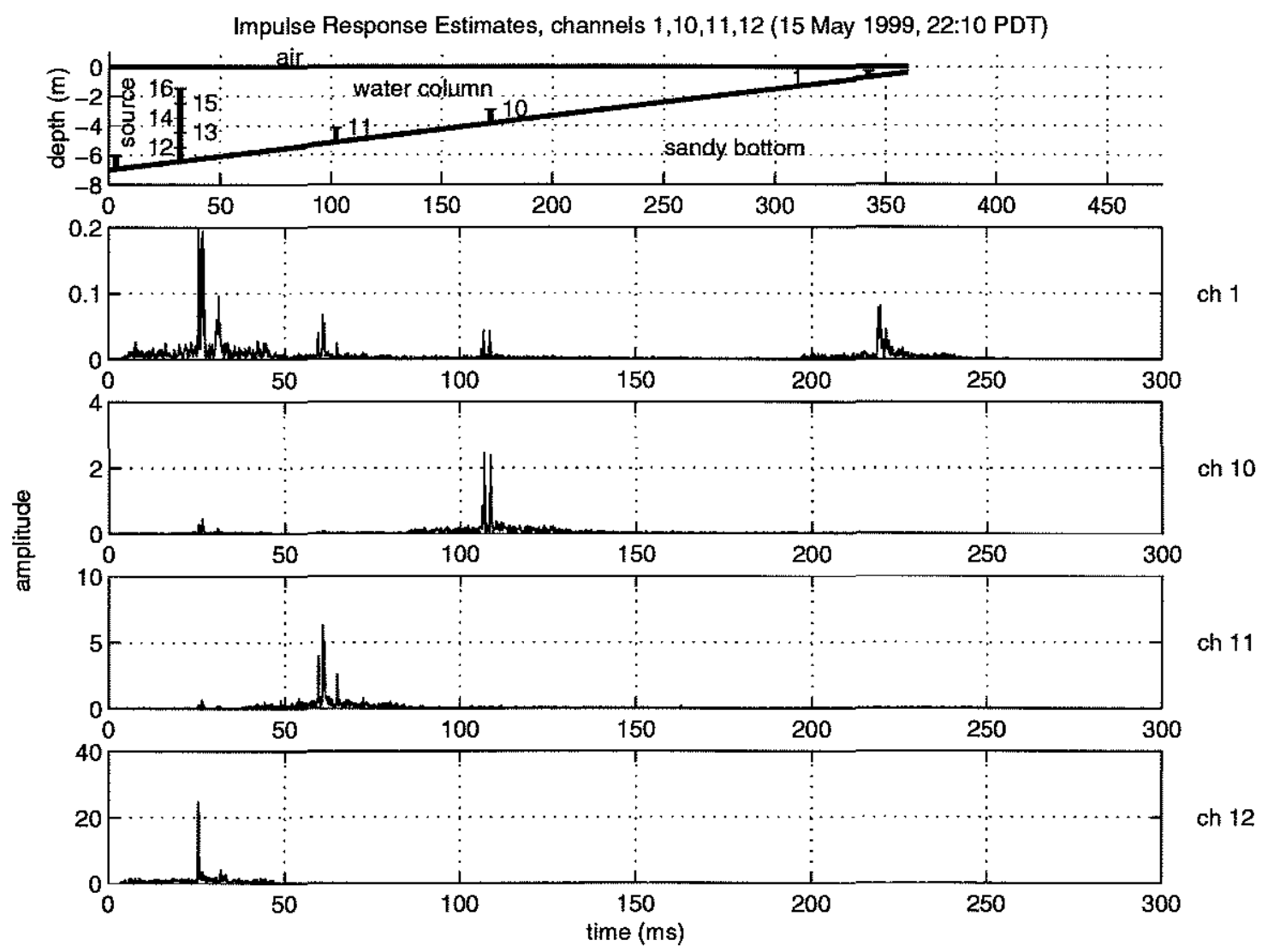

Figure 2-1: The top plot shows the Scripps Pier geometry, with the hydrophone locations labelled by hydrophone number, and the horizontal axis in meters. The lower four plots show the impulse response estimates on hydrophones $1,10,11$, and 12, when transmitting from deep-water source 4 . Note the different amplitude scales. 
channels where the hydrophone was distant from the source. The crosstalk problem was most severe for hydrophones distant from the transmitting source, since the recorded signal was dominated by the crosstalk.

With the fairly strong crosstalk which existed, signals which were longer than the propagation delay of the channel were mixed together. Some of the data could be processed to yield estimates of the channel impulse responses. None of the data containing crosstalk, however, is useful for testing communication algorithms or performance. The acoustic propagation delay for the entire channel, covering about $300 \mathrm{~m}$, was about $200 \mathrm{~ms}$. Between neighboring hydrophones, however, a typical propagation delay was about $50 \mathrm{~ms}$, corresponding to roughly $70 \mathrm{~m}$. Signals longer than these propagation delays, and also continuously repeated signals, would have been mixed together by the crosstalk, as an inshore hydrophone would simultaneously receive an acoustic signal and a crosstalk signal from a later part of the transmitted sequence, coupled in from a deep-water hydrophone.

\subsection{Crosstalk Model}

The crosstalk was caused by a wiring mistake in the hydrophone cable. A shielded pair of cables carried the signal from each hydrophone back to the hydrophone cable breakout box, the preamplifier, and the recorder. Rather than being connected together and tied to a salt water ground, the individual shields were connected to the negative input on each channel. The shields lay next to one another in the hydrophone cable for as much as $300 \mathrm{~m}$, and the capacitive coupling between the shields, directly connected to the negative input of each channel, led to the crosstalk.

An AC circuit model of a pair of channels, including hydrophones, breakout box, and preamplifier, is shown in Figure 2-2. The hydrophones, which were used in current mode, are modeled as voltage sources in series with a resistor; equivalently, they could be modeled as current sources in parallel with a resistor. The capacitive coupling between channels $i$ and $j$ is shown by the capacitor $C_{i j}$. In the frequency bands of interest, $9-14 \mathrm{kHz}$, the preamplifier filters, $G(\omega)$, were essentially constant, with $20 \mathrm{~dB}$ of gain.

The circuit can be analyzed through superposition. When there is no voltage on hydrophone $i$, the entire signal at the output of channel $j, v_{j o}$, is due to the voltage $v_{j}$ across the hydrophone 


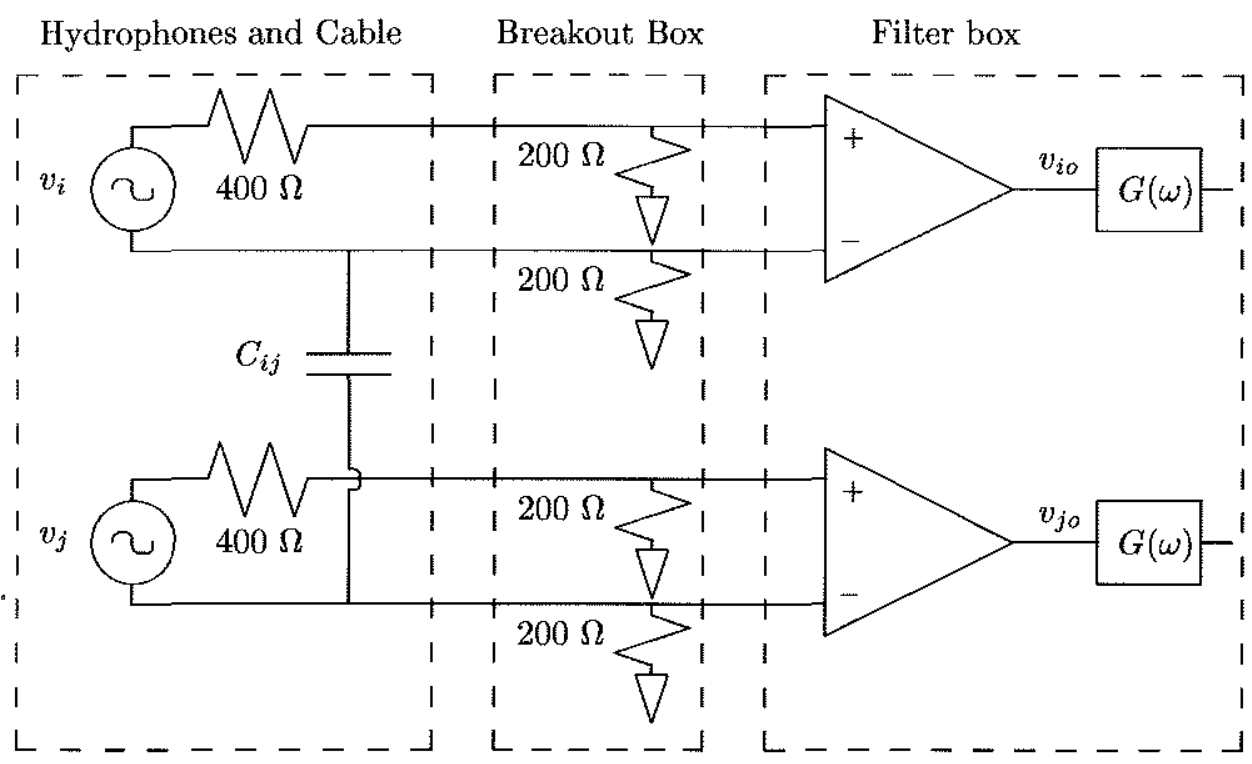

Figure 2-2: An AC circuit model of the hydrophones and hydrophone cable, breakout box, and filter box. The shield-to-shield capacitance $C_{i j}$ couples channels $i$ and $j$.

on channel $j$. The current flowing in channel $j$ 's resistor network is then $I=v_{j} / 800 \Omega$, since the instrumentation amplifiers in the preamplifier draw negligible current. Simplifying the circuit for this case, the voltage between the positive and negative inputs of the instrumentation amplifier is $v_{j o}=400 \Omega \cdot I=\frac{1}{2} v_{j}$.

When there is no voltage on hydrophone $j$, the output on channel $j$ is driven by the capacitive coupling from channel $i$. Assuming that the current flowing through the coupling capacitor $C_{i j}$ can be neglected, the voltage on the capacitor is $-v_{i} / 4$, by an analysis similar to that above. Again using the fact that the current drawn by the instrumentation amplifier inputs can be neglected, the current flowing from the coupling capacitor to the positive amplifier input flows through $600 \Omega$ to ground; the current to the negative input flows through $200 \Omega$ to ground. These paths are in parallel, so the voltage difference at the amplifier inputs is

$$
v_{j o}=-\frac{1}{2} \frac{200 \Omega}{Z_{C_{i j}}+200 \Omega \| 600 \Omega}\left(-\frac{v_{i}}{4}\right)
$$

where the impedance of the capacitor is $Z_{C_{i j}}=1 / i \omega C_{i j}$, and $\|$ indicates parallel connection of components.

Using superposition, the output voltage from preamplifier $j$, the signal which was then recorded 
directly, is

$$
v_{o j}=\frac{1}{2} v_{j}+\frac{1}{8} \sum_{i \neq j} \frac{200 \Omega}{Z_{C_{i j}}+200 \Omega \| 600 \Omega} v_{i}=\frac{1}{2} v_{j}+\sum_{i \neq j} \frac{\alpha_{i j}}{2} v_{i},
$$

where $\alpha_{i j}$ is the crosstalk coupling coefficient between channels $i$ and $j$, and the factor of $1 / 2$ is due to the definitions of the input and output voltages, since without crosstalk, $v_{i o}=\frac{1}{2} v_{o}$.

Finally, the assumption that the current flowing in the coupling capacitor can be neglected compared with the current flowing directly in the resistor networks needs to be checked. The measured crosstalk coefficients have magnitudes of roughly $-35 \mathrm{~dB}$ to $-40 \mathrm{~dB}$, at a carrier frequency of $11.5 \mathrm{kHz}$. Using the model above, this gives a coupling capacitance value of around $3 \mathrm{nF}$, which is consistent with typical conductor-to-conductor capacitances over several hundred meters of cable. ${ }^{1}$ The model gives plausible predictions for the coupling capacitance, and at these capacitances, the current through the coupling capacitor is less than $5 \%$ of the total current flowing in the resistor network, so the approximations made above are valid.

The circuit model above can be further reduced to an effective high-pass filter coupling the charmels, shown in Figure 2-3a. The effective resistance is $R_{\mathrm{eff}}=150 \Omega$, and the capacitance is the coupling capacitance, $C_{i j}$, estimated to be $1 \mathrm{nF}$ to $10 \mathrm{nF}$. The characteristic time constant for this circuit is then on the order of $1 \mu \mathrm{s}$ or less. The transfer function of this system is

$$
H(\omega)=\frac{i \omega R_{\mathrm{eff}} C_{i j}}{1+i \omega R_{\mathrm{eff}} C_{i j}}=i \omega R_{\mathrm{eff}} C_{i j}\left(1-i \omega R_{\mathrm{eff}} C_{i j}+\mathcal{O}\left(\omega^{2} R_{\mathrm{eff}}^{2} C_{i j}^{2}\right)\right)
$$

and its magnitude and phase are plotted in Figure 2-3b, for $C_{i j}=10 \mathrm{nF}$, which has the slowest rise time for our range of interest in $C_{i j}$. Over the frequency band of interest, $9-14 \mathrm{kHz}$, the phase is nearly constant, and the magnitude is a linear function of frequency.

\subsection{Crosstalk Correction}

With a model of the crosstalk, the next step is to estimate the crosstalk coefficients, and correct the recorded signals.

\footnotetext{
${ }^{1}$ Datasonics measured the coupling capacitances in the cable after the experiment, and came up with typical values of $30 \mathrm{nF}$ to $90 \mathrm{nF}$ (see Table 2-2). These values would give crosstalk coefficients on the order of $-20 \mathrm{~dB}$ to $-12 \mathrm{~dB}$ in this model, which are much stronger than the observed crosstalk. The resistor network in the breakout box is well-known, and the error causing the crosstalk is well-known, so it is likely that the actual coupling capacitances were somewhat smaller, in the range of $1 \mathrm{nF}$ to $10 \mathrm{nF}$.
} 

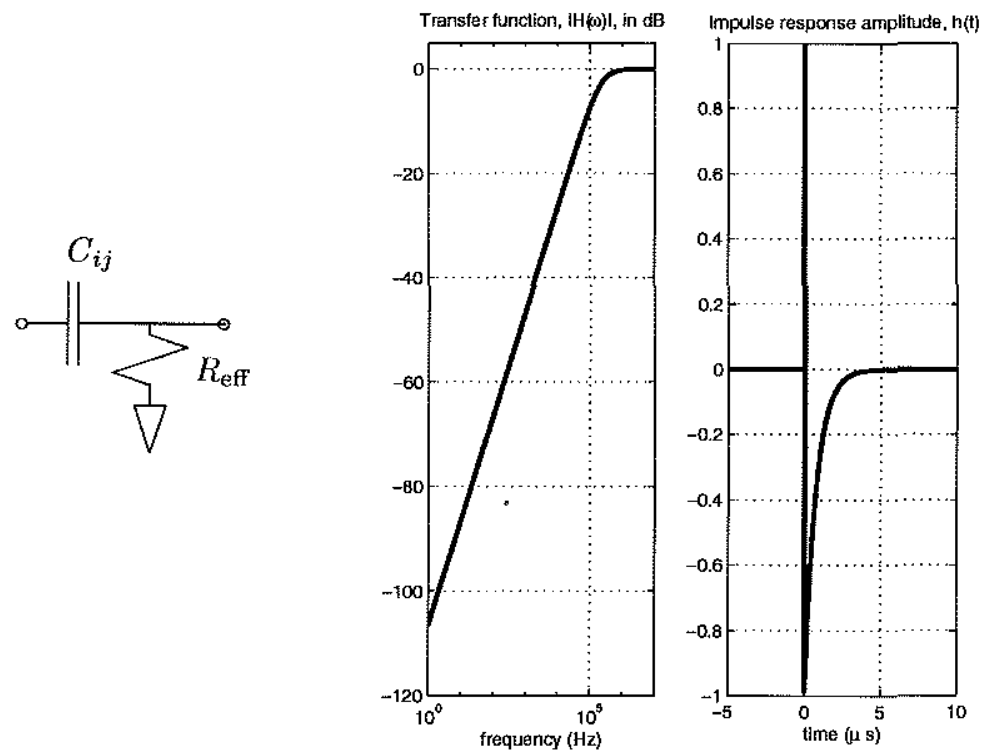

Figure 2-3: (a) The effective high-pass filter, (b) its transfer function, and (c) its impulse response.

The primary channel characterization signals which were transmitted were length-511 binary phaseshift key (BPSK) maximal-length pseudo-random sequences (M-sequences). When an M-sequence is cross-correlated against a repeated train of the same M-sequence, the result approximates a train of impulses, with low sidelobes. In channel characterization experiments, a train M-sequences is transmitted through the acoustic communication channel. The recorded signal is the convolution of the M-sequence train with the channel impulse response. Cross-correlating the recorded signal with the transmitted $\mathrm{M}$-sequence yields a series of channel impulse response estimates, which track the evolution of the time-variant channel[16].

Due to the crosstalk mixing in the Scripps Pier experiment, the cross-correlation of the baseband data for a given channel will not directly yield an impulse response estimate for that channel, but rather a superposition of the impulse response estimates for all the channels. Once crosstalk was determined to be a significant problem, additional characterization probes were added, namely short length- 88 Turyn codes. Cross-correlation of isolated length- 88 Turyn codes also yields an impulse response estimate, with a peak-to-maximum-sidelobe amplitude ratio of $88 / 5$, or about $25 \mathrm{~dB}[12]$. The cross-correlation of length-511 $\mathrm{M}$-sequences has a peak-to-sidelobe amplitude ratio of 511 , or $54 \mathrm{~dB}$, so almost $30 \mathrm{~dB}$ of peak-to-sidelobe power was lost by switching to length- 88 Turyn codes[16]. Due to their short length, however, $22 \mathrm{~ms}$ at 4000 symbols/sec, these Turyn codes can be range-gated at each hydrophone, and by doing that, individual, uncontaminated estimates 
of the crosstalk coefficients can be made for each channel.

If the crosstalk coefficients are constant over time, then the bulk of the data, including the long sections of repeated M-sequences, can be pre-corrected for crosstalk, then processed as usual for channel characterization. This is especially important because of the simultaneous environmental measurements made by the teams from Scripps Institution of Oceanography (SIO) and Institute of Ocean Sciences (IOS). Combining these measurements with the extensive acoustic channel probes creates a potentially very valuable data set for correlating the environmental conditions to the acoustic communication channel parameters which they drive.

\section{Coefficient Estimation}

To analyze the crosstalk coefficient estimation, let $s_{j}[k]$ be the baseband signal received acoustically at hydrophone $j$ at time $k$, from a source transmitting the pseudo-random noise sequence $s_{o}[k]$. Similarly, let $r_{j}[k]$ be the baseband signal recorded on channel $j$ (i.e. without matched filtering). With crosstalk, the signal recorded from hydrophone $j$ can be modeled as a linear combination of the acoustically-received signal at each hydrophone, and zero-mean white noise with variance $\sigma^{2}$, $n[k]$ :

$$
r_{j}[k]=s_{j}[k]+\sum_{i \neq j} \alpha_{i} s_{i}[k]+n[k]
$$

The $\alpha_{i}$ are unknown dimensionless crosstalk coefficients to be estimated for coupling channels $i$ to channel $j$. As shown above, it is sufficient to consider frequency-independent crosstalk coupling, i.e. a single coefficient per channel.

In the regions where the crosstalk coefficients are to be estimated through range-gating, there is essentially no real signal in $s_{j}[k]$; it can therefore be combined into the noise term, $n[k]$. Since the acoustically-received signals $s_{i}[k]$ were not preserved directly, they can be approximated as the baseband recorded signals $r_{i}[k]$, which is a reasonable approximation. The model then reduces to

$$
r_{j}[k]=\sum_{i \neq j} \alpha_{i} r_{i}[k]+n[k]
$$

This model can then be written as $R \underline{\alpha}+\mathbf{n}=\mathbf{r}_{j}$, where $R$ is a matrix formed of the baseband recorded signals $\mathbf{r}_{i}$, taken as column vectors, and $\mathbf{r}_{j}$ and $\mathbf{n}$ are the recorded signal and effective additive noise, respectively, again taken as column vectors. Underlining or bold face indicates vector quantities. Equivalently, the model could be written in terms of the impulse response estimates of 
the channel, $h_{i}[n]$, rather than the baseband recorded signals, $r_{i}[n]$, since a linear transformation relates the two, namely cross-correlation with the transmitted signal.

In the model $R \underline{\alpha}+\mathbf{n}=\mathbf{r}_{j}$, the noise vector $\mathbf{n}$ is unknown. The linear least-squares estimate of $\underline{\alpha}$ (using the baseband recorded signal in $R$ and $\mathbf{r}_{j}$ ) is denoted by $\underline{\hat{\alpha}}_{r}$, and is the estimate which minimizes $\left|R \underline{\alpha}_{r}-\mathbf{r}_{j}\right|^{2}$. The linear least-squares estimate is

$$
\underline{\hat{\alpha}}_{r}=\left(R^{H} R\right)^{-1} R^{H} \mathbf{r}_{j}=\underline{\alpha}+\left(R^{H} R\right)^{-1} R^{H} \mathbf{n} .
$$

\section{Variance of coefficients, baseband signals versus impulse response esti- mates}

The coefficient estimates can be made either with the baseband signals, or with the impulse response estimates resulting from matched-filtering the baseband signals with the baseband transmitted waveform. The method producing more robust estimates will be preferable.

From the previous section, the model for the crosstalk is

$$
R \underline{\alpha}+\mathbf{n}=\mathbf{r}_{j}
$$

In this expression, $R$ is a matrix, and its columns are the recorded signals from the channels which dominate the crosstalk (offshore channels 10-15). The recorded signal on an inshore hydrophone (channels 1-6) is represented by the column vector $\mathbf{r}_{j}$. In the crosstalk model, during the time before the acoustic signal has propagated to the inshore hydrophones, the only contributions to the received signal $\mathbf{r}_{j}$ are noise and crosstalk. The column vector $\mathbf{n}$ is a zero-mean white noise vector. The crosstalk is expressed as the product between $R$ and $\underline{\alpha}$. In the case of a single-tap crosstalk model, where a length-1 filter, or constant gain term, represents the crosstalk transfer function, $\underline{\alpha}$ is a column vector of crosstalk coefficients between all of the offshore channels (channels 10-15) and the inshore channel to be corrected (only one of channels 1-6). For multiple-tap crosstalk models, $\underline{\alpha}$ would be a matrix with as many columns as there were taps in the model. For simplicity, the remainder of this section will be restricted to single-tap models.

The linear least-squares estimate of $\underline{\alpha}$ is

$$
\underline{\hat{\alpha}}_{r}=\underline{\alpha}+\left(R^{H} R\right)^{-1} R^{H} \mathbf{n},
$$


as shown in the previous section. The matrix $R$ can be written as $R=R_{o}+N$, where $R_{o}$ is a matrix of the received signals due to deterministic propagation through the channel at a particular instant, and $N$ is a noise matrix containing the received noise column vectors for each channel. The noise at any pair of hydrophones is uncorrelated, and furthermore it is modeled as zero-mean white noise. Because of these assumptions, $N$ and $\mathbf{n}$ are uncorrelated. The expectation value of $\underline{\hat{\alpha}}_{r}$ is

$$
\begin{aligned}
E\left[\underline{\hat{\alpha}}_{r}\right] & =\underline{\alpha}+E\left[\left(R^{H} R\right)^{-1} R^{H} \mathbf{n}\right] \\
& =\underline{\alpha}+E\left[\left(R^{H} R\right)^{-1} R^{H}\right] E[\mathbf{n}]=\underline{\alpha},
\end{aligned}
$$

since $N$ (as an additive component of $R$ ) and $\mathbf{n}$ are uncorrelated, and $\mathbf{n}$ is zero-mean. Therefore, $\underline{\hat{\alpha}}_{r}$ is an unbiased estimator of $\underline{\alpha}$.

Now consider filtering the baseband signal with a matched filter. For the transmitted signal $s_{o}[k]$, with energy $\mathcal{E}_{s}$, the matched filter is $s_{o}[-k] / \mathcal{E}_{s}$. Defining the matched-filtered data as $m_{j}[k]=r_{j}[k] * s_{o}[-k] / \mathcal{E}_{s}$, and applying the matched filter to Equation 2.1 yields

$$
m_{j}[k]=\sum_{i \neq j} \alpha_{i} m_{i}[k]+n^{\prime}[k]
$$

In this expression, the noise vector, $\mathbf{n}^{\prime}$, is still approximately a white noise, zero-mean vector, since the matched filter consists of a pseudorandom sequence and provides little shaping. This can be written in matrix form as

$$
M \underline{\alpha}+\mathbf{n}^{\prime}=\mathbf{m}_{j}
$$

where $M$ is a matrix of the column vectors $\mathbf{m}_{i}$. This form is directly analogous to Equation 2.2 . Following the derivation of Equation 2.3, the linear least-squares estimate of $\underline{\alpha}$ using the matchedfilter data, $\underline{\hat{\alpha}}_{m}$, is

$$
\underline{\hat{\hat{\alpha}}}_{m}=\underline{\alpha}+\left(M^{H} M\right)^{-1} M^{H} \mathbf{n}^{\prime} .
$$

As with the estimator in the unfiltered baseband case, $\underline{\hat{\alpha}}_{m}$ is an unbiased estimator of $\underline{\alpha}$.

Since estimators of the crosstalk coefficients in both the unfiltered and matched-filtered cases are unbiased estimators, the variance of the estimators will determine which estimator has a lower expected error; the one with the lower variance will have the lower expected estimation error. 
Returning to the unfiltered baseband signal case, the covariance of the estimator is

$$
\operatorname{Cov}\left(\underline{\hat{\alpha}}_{r}\right)=E\left[\underline{\underline{\hat{\alpha}}}_{r} \underline{\hat{\hat{\alpha}}}_{r}^{H}\right]-E\left[\underline{\hat{\hat{\alpha}}}_{r}\right] E\left[\underline{\hat{\hat{\alpha}}}_{r}^{H}\right]
$$

From above, $E\left[\underline{\hat{\alpha}}_{r}\right]=\underline{\alpha}$. The remaining term in the covariance is

$$
E\left[\underline{\hat{\alpha}}_{r} \underline{\hat{\alpha}}_{r}^{H}\right]=\underline{\alpha \alpha}^{H}+E\left[\left(R^{H} R\right)^{-1} R^{H} \mathbf{n n}^{H} R\left(R^{H} R\right)^{-1}\right] .
$$

The crossterms vanish because they have expected value zero, due to $N$ and $\mathbf{n}$ being uncorrelated.

The expected value in this expression can be evaluated with an iterated expectation:

$$
\begin{aligned}
E\left[\left(R^{H} R\right)^{-1} R^{H} \mathbf{n n}^{H} R\left(R^{H} R\right)^{-1}\right] & =E_{R}\left[E_{n \mid R}\left[\left(R^{H} R\right)^{-1} R^{H} \mathbf{n} \mathbf{n}^{H} R\left(R^{H} R\right)^{-1} \mid R\right]\right] \\
& =\sigma^{2} E\left[\left(R^{H} R\right)^{-1} R^{H} R\left(R^{H} R\right)^{-1}\right] \\
& =\sigma^{2} E\left[\left(R^{H} R\right)^{-1}\right]
\end{aligned}
$$

Therefore the covariance of the estimator is

$$
\operatorname{Cov}\left(\underline{\hat{\alpha}}_{r}\right)=\sigma^{2} E\left[\left(R^{H} R\right)^{-1}\right]
$$

To evaluate the expectation value in the estimator's covariance, several approximations are necessary. Expanding the term $R^{H} R$ yields

$$
R^{H} R=R_{o}^{H} R_{o}+R_{o}^{H} N+N^{H} R_{o}+N^{H} N
$$

If the levels in the noise matrix $N$ are small compared with the signal levels in $R_{o}$, or if the signals in the columns of $R_{o}$ are fairly variable, then the crossterms will be small, and can be neglected. Furthermore, if the signals in the columns of $R_{o}$ have little overlap, then $\left(R_{o}^{H} R_{o}\right)_{i j} \approx \mathcal{E}_{i} \delta_{i j}$, where $\mathcal{E}_{i}$ is the energy in signal $\mathbf{r}_{i}$. Finally, if $N^{H} N$ within the expected value can be replaced with $E\left[N^{H} N\right]=\sigma^{2} I$, then

$$
\begin{aligned}
\operatorname{Cov}\left(\underline{\hat{\alpha}}_{r}\right) & =\sigma^{2} E\left[\left(R^{H} R\right)^{-1}\right] \\
\operatorname{Cov}\left(\underline{\hat{\alpha}}_{r}\right) & \approx \sigma^{2} E\left[\left(I+\left(R_{o}^{H} R_{o}\right)^{-1} N^{H} N\right)^{-1}\left(R^{H} R\right)^{-1}\right] \\
\operatorname{Cov}\left(\underline{\hat{\alpha}}_{r}\right)_{i j} & \approx \frac{\sigma^{2}}{\mathcal{E}_{i}+\sigma^{2}} \delta_{i j}
\end{aligned}
$$


In the case where the matched-filtered impulse response estimates are used instead of the baseband signals, the derivation is identical, except the noise variance is different. The filtered noise vector $\mathbf{n}^{\prime}$ is formed from the white noise sequence $n[k]$, filtered with the matched filter, $s_{o}[-k] / \mathcal{E}_{s}$. The matched filter is a Turyn code of length $K=88$, and each sample has amplitude $\pm A$. Since $n[k]$ is a sequence of independent, identically-distributed samples with variance $\sigma^{2}$, the variance of $\mathbf{n}^{\prime}$ is

$$
\operatorname{Var}\left(\mathbf{n}^{\prime}\right)=\sigma^{2} \frac{K A^{2}}{\mathcal{E}_{s}^{2}}=\frac{\sigma^{2}}{\mathcal{E}_{s}}
$$

The covariance of the crosstalk coefficient estimator, in the case of matched-filtered signals, is then

$$
\operatorname{Cov}\left(\underline{\hat{\alpha}}_{m}\right)_{i j} \approx \frac{\sigma^{2}}{\mathcal{E}_{i} \mathcal{E}_{s}+\sigma^{2}} \delta_{i j}<\operatorname{Cov}\left(\underline{\hat{\alpha}}_{r}\right)_{i j}
$$

The energy $\mathcal{E}_{s}$ in the Turyn code is greater than unity in this normalization, so the coefficient estimates from the matched-filtered signals have lower variances than the estimates from the signals which are basebanded only. Since both estimators are unbiased estimators of the crosstalk coefficients $\alpha$, the estimator using matched-filtered signals will have a lower expected estimation error. This is the principal advantage of using the matched-filtered signals instead of the baseband signals. A secondary advantage of compressing the signal energy into impulse response estimates is that the impulse response estimates are easier to range-gate than the unfiltered baseband signals are. Finally, since the expected value of both estimators is the same, the crosstalk coefficient estimates from the matched-filtered impulse responses can be used to correct the unfiltered, baseband signals.

If the coefficients are stable in time, low-variance coefficient estimates obtained from the matchedfiltered data using Turyn codes can be applied to other signals, including data from continuous M-sequences, where simple crosstalk coefficient estimates are impossible due to the inability to range-gate the signals.

\section{Number of Taps}

In order to correct the sampled data for crosstalk, a discrete-time model of the continuous-time crosstalk coupling must be made. The simplest approach is to model the coupling with finite impulse response (FIR) discrete-time transfer functions between the various channels. The convolutions resulting from FIR filtering can be immediately formulated as a linear least squares problem to estimate the tap values. 
The continuous-time model derived above is explicitly frequency-dependent, since the coupling mechanism is a high-pass filter. Ideally, the discrete-time filter will be frequency-dependent as well, to capture the coupling most accurately. The simplest frequency-dependent FIR filter has two taps; a single-tap filter is just a constant gain. The energy residual from linear least-squares correction with two taps (parameters) will by definition be as small or smaller than with a single tap, for a given section of data.

With data from tape 1352210, when linear least-squares corrections were made by estimating the tap values for each impulse response estimate separately, the correction with two taps was somewhat better than with a single tap. With a single tap, the mean energy residual in the crosstalk region (relative to the crosstalk energy before correction) was $-9.5 \mathrm{~dB}$ versus $-12.0 \mathrm{~dB}$ for two taps. Typical values for the tap coefficients are shown in Table 2-1.

The coefficients for the second tap in the two-tap filters are not stable in time, however. Some insight into this problem can be gained by considering sampling the impulse response of the continuous-time filter, plotted in Figure 2-3c. The impulse response has almost completely returned to zero after roughly $5 \mu \mathrm{s}$. The sampling rate of the system was only $48 \mathrm{kHz}$, however, corresponding to a sampling period of $20.8 \mu \mathrm{s}$. Since the impulse response of the effective high-pass filter is short compared with the sampling period of the data, the estimate of the second tap of a two-tap filter may be dominated by noise.

Considering the continuous time transfer function also indicates that the second tap may be heavily influenced by noise. Letting $\omega=\omega_{c}+\tilde{\omega}$, and expanding the transfer function for small perturbations from the carrier frequency $\omega_{c}$,

$$
H(\tilde{\omega}) \approx\left(i \omega_{c} R_{\mathrm{eff}} C_{i j}+\omega_{c}^{2} R_{\mathrm{eff}}^{2} C_{i j}^{2}\right)+\tilde{\omega}\left(i R_{\mathrm{eff}} C_{i j}+2 \omega_{\mathrm{c}} R_{\mathrm{eff}}^{2} C_{i j}^{2}\right)=A+\tilde{\omega} B
$$

With typical values for $R_{\text {eff }}$ and $C_{i j}, A$ is approximately $-30 \mathrm{~dB}$, and $B$ is approximately $-125 \mathrm{~dB}$. Any small amount of noise in the data will make $B$, corresponding to the second tap in a two-tap filter, extremely difficult to estimate reliably. 


\section{Single Coefficient Estimates versus Continuous Estimates}

Although the energy residuals for the crosstalk correction using a two-tap filter mentioned in the previous section were slightly smaller than the residuals when using a single tap, these results were for cases where it was possible to re-estimate the tap coefficients at each impulse response. The short, isolated Turyn codes allow the crosstalk from individual channels to be identified, due to acoustic propagation delays. Continuous maximal-length sequences, however, formed the majority of the channel probe transmissions, and cannot be range-gated to estimate crosstalk coefficients in a straightforward manner.

To correct the crosstalk on the maximal-length sequences, it is therefore difficult to estimate the crosstalk coefficients for each impulse response. Instead, the crosstalk coefficients must be estimated from a section of data which can be range-gated, such as a section of Turyn code transmissions, then those coefficients must be applied to the rest of the data, without further coefficient updates.

In correcting the data from tape 1352210, when the tap values were estimated from the first 49 impulse response estimates, and applied in the crosstalk correction of all later impulse response estimates, the two-tap correction was still better, but not as markedly so as in the single-tap case. With a single tap, the mean energy residual using a single initial coefficient estimate was $-8.8 \mathrm{~dB}$, and $-9.3 \mathrm{~dB}$ for two-tap correction. Furthermore, these initial coefficient estimates were applied to data which was separated by at most two hours. If the tap values drift over timescales larger than an hour, the single-estimate case will end up becoming significantly worse than the tracking case, where a new estimate of the tap coefficients is made for every impulse response estimate. For timescales of up to an hour or two, however, the case with a single initial estimate did not become increasingly worse with time, when compared with the tracking case; rather, it remained at a level several $\mathrm{dB}$ worse than the tracking case. Figure 2-4 shows a typical sequence for the energy residuals for both the single-estimate and tracking cases.

Even though no significant long-term drift in the tap coefficients was observed, the linear leastsquares estimates of their values do fluctuate significantly over short timescales, such as seconds or minutes. Because the coefficients in the second tap case are much less stable over time than the single-tap crosstalk coefficients, a single-tap, frequency-independent crosstalk correction method would be more reliable for correcting most of the data. 


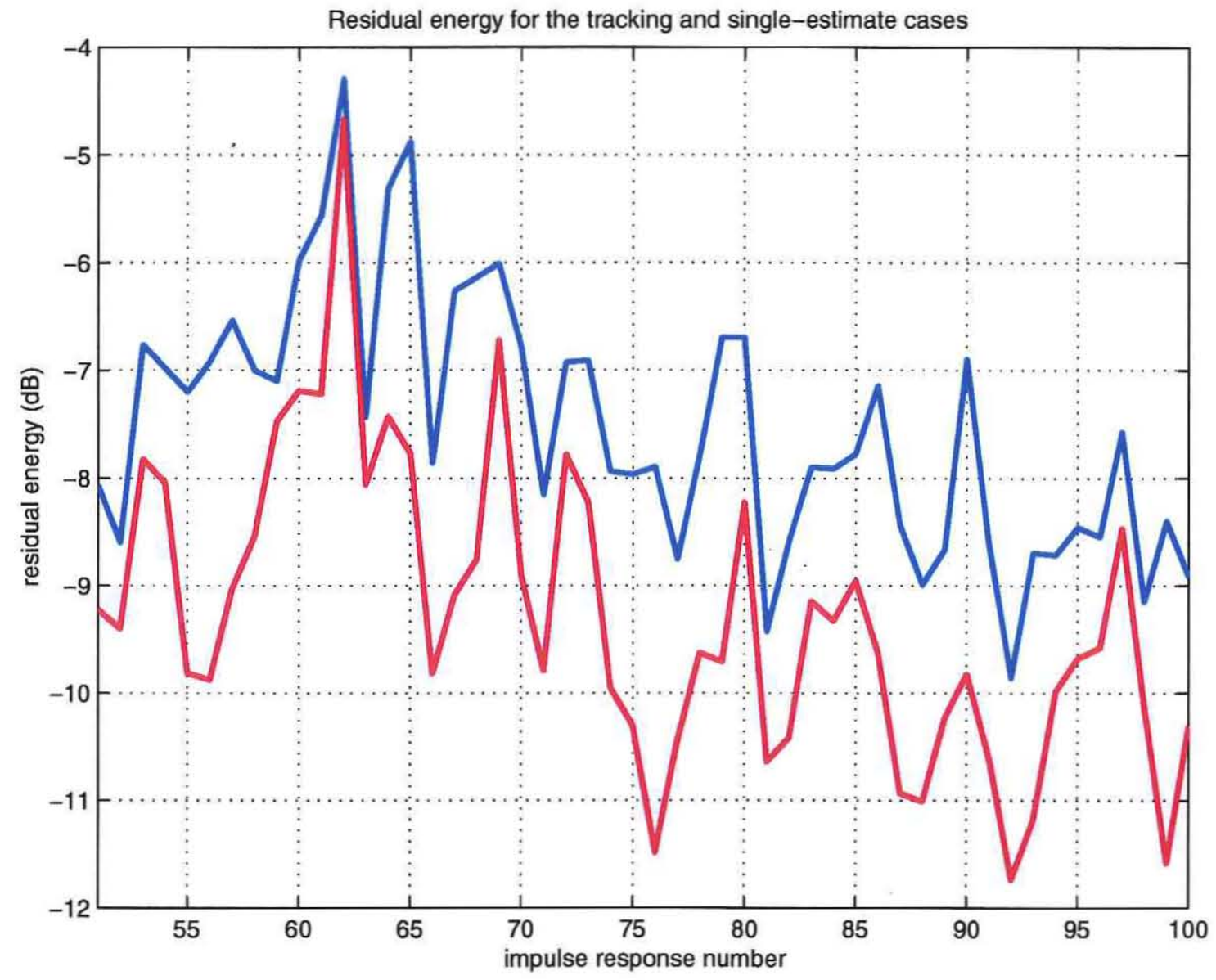

Figure 2-4: Plotted in blue is the residual energy after crosstalk correction using an initial estimate of the crosstalk coefficients (from the first 49 impulse responses), applied to all later data. Plotted in red is the residual energy after correction when the crosstalk coefficients were estimated for every impulse response. Both curves show the residuals for impulse responses 50-100. The residuals for the case with an initial estimate are typically $3-4 \mathrm{~dB}$ worse than those for the tracking estimates, even up to two hours after the initial estimates were made. 
The variability in the tap estimates is primarily due to fuctuations in phase, rather than magnitude. As shown in Table 2-1, in both the one-tap and two-tap cases, the phases of the coefficients have a large standard deviation relative to their mean values, whereas the magnitudes have a small standard deviation relative to their mean values. The standard deviations in both phase and magnitude are significantly larger for channels 13-15 compared with 10-11. Channel 12 in most cases has a larger standard deviation than channels $10-11$, but not always as large as channels 13-15, which is surprising, since it is in the same vertical array and should have similar problems due to channel 16 .

\begin{tabular}{|l||c|c|c|c|c|c|c|}
\hline \hline \multicolumn{7}{|c|}{ Table 2-1: Tap Coefficients } \\
\hline \hline Channel & 10 & 11 & 12 & 13 & 14 & 15 \\
\hline \multicolumn{7}{|c|}{ One-tap coefficients } \\
\hline \hline Magnitude mean & 0.0174 & 0.0102 & 0.0112 & 0.0055 & 0.0136 & 0.0063 \\
\hline Magnitude st. dev. & 0.0005 & 0.0003 & 0.0012 & 0.0008 & 0.0011 & 0.0014 \\
\hline Angle mean & -0.3164 & -0.1164 & -0.8809 & -0.2180 & -0.9107 & 0.1138 \\
\hline Angle st. dev. & 0.0334 & 0.0318 & 0.1151 & 0.1476 & 0.0786 & 0.2258 \\
\hline & Two-tap coefficients & & \\
\hline \hline Magnitude mean (tap 1) & 0.0169 & 0.0097 & 0.0111 & 0.0056 & 0.0125 & 0.0072 \\
\hline Magnitude mean (tap 2) & 0.0016 & 0.0010 & 0.0022 & 0.0008 & 0.0034 & 0.0036 \\
\hline Magnitude st. dev. (tap 1) & 0.0006 & 0.0003 & 0.0012 & 0.0008 & 0.0010 & 0.0014 \\
\hline Magnitude st. dev. (tap 2) & 0.0006 & 0.0002 & 0.0006 & 0.0004 & 0.0016 & 0.0009 \\
\hline Angle mean (tap 1) & -0.2794 & -0.1007 & -0.9595 & -0.1375 & -0.9468 & 0.1522 \\
\hline Angle mean (tap 2) & -1.1586 & -0.3605 & -0.4354 & 0.2283 & -0.3394 & -0.5333 \\
\hline
\end{tabular}

\subsection{Results}

\section{Transmissions from outer source}

For the data which was transmitted from deep-water source 4, the most important hydrophone channels are the hydrophones relatively close to shore, namely channels 1-10. These are the hydrophones which are surrounded at times by bubble clouds, and determining their availability for communication data links was a major goal of the experiment. These are also the channels, however, where crosstalk was most significant when transmitting from the outer sources, because the acoustically-received energy was in general less than the energy received from crosstalk. 
In this transmission geometry, the vertical hydrophone array, channels $12-16$, is very close to the source, and crosstalk is negligible, so the recorded signal on these channels can be taken to be the acoustically-received signal. Further inshore, channels 10 and 11 have crosstalk contributions from channels 12-16, but very little crosstalk contribution from the inner hydrophones, channels 1-9. The crosstalk on these innermost phones is significant, often stronger than the acoustically-received signal, and is dominated by contributions from channels $12-16,11$, and 10 , with only a small amount of channel-to-channel crosstalk amongst the innermost hydrophones.

With this crosstalk structure, an iterative crosstalk correction procedure can be developed. Channels 12-15 are left uncorrected. (Channel 16 was not recorded, but its hydrophone remained powered during the experiment. This problem will be discussed further below.) For a given inshore channel, the crosstalk coefficients coupling that channel to channels 12-15 are calculated in a small range-gated region of the impulse response estimate. The entire impulse response estimates for 12-15, weighted by the coefficient estimates, are then subtracted from the impulse response estimate for the inshore channel.

Next, the impulse response estimate for channel 11 is corrected for channels 12-15 in the same manner: the crosstalk coefficients between 11 and 12-15 are estimated in a range-gated region, and the entire impulse response estimates for 12-15, each weighted by a constant, are subtracted from channel 11. A crosstalk coefficient is estimated between channel 11 and the inshore channel, and the inshore channel is corrected for crosstalk from 11. This is repeated for channel 10, this time correcting channel 10 for channel 11 , as well as $12-15$, before correcting the inshore channel for crosstalk from 10.

At this point, the inshore hydrophone channel has been corrected for crosstalk from all the outer hydrophones which were recorded. This removes the vast majority of the crosstalk energy contributed to the inshore channel, due to the high acoustically-received signal levels on the outer channels. This process is shown in Figure 2-5.

\section{Residual energy}

As shown for a single set of impulse responses in Figure 2-5, crosstalk correction using singleparameter linear least squares fitting works fairly well. The energy due to crosstalk from channels 10 and 11 can be largely removed from inshore hydrophone channels, and most of the energy due 


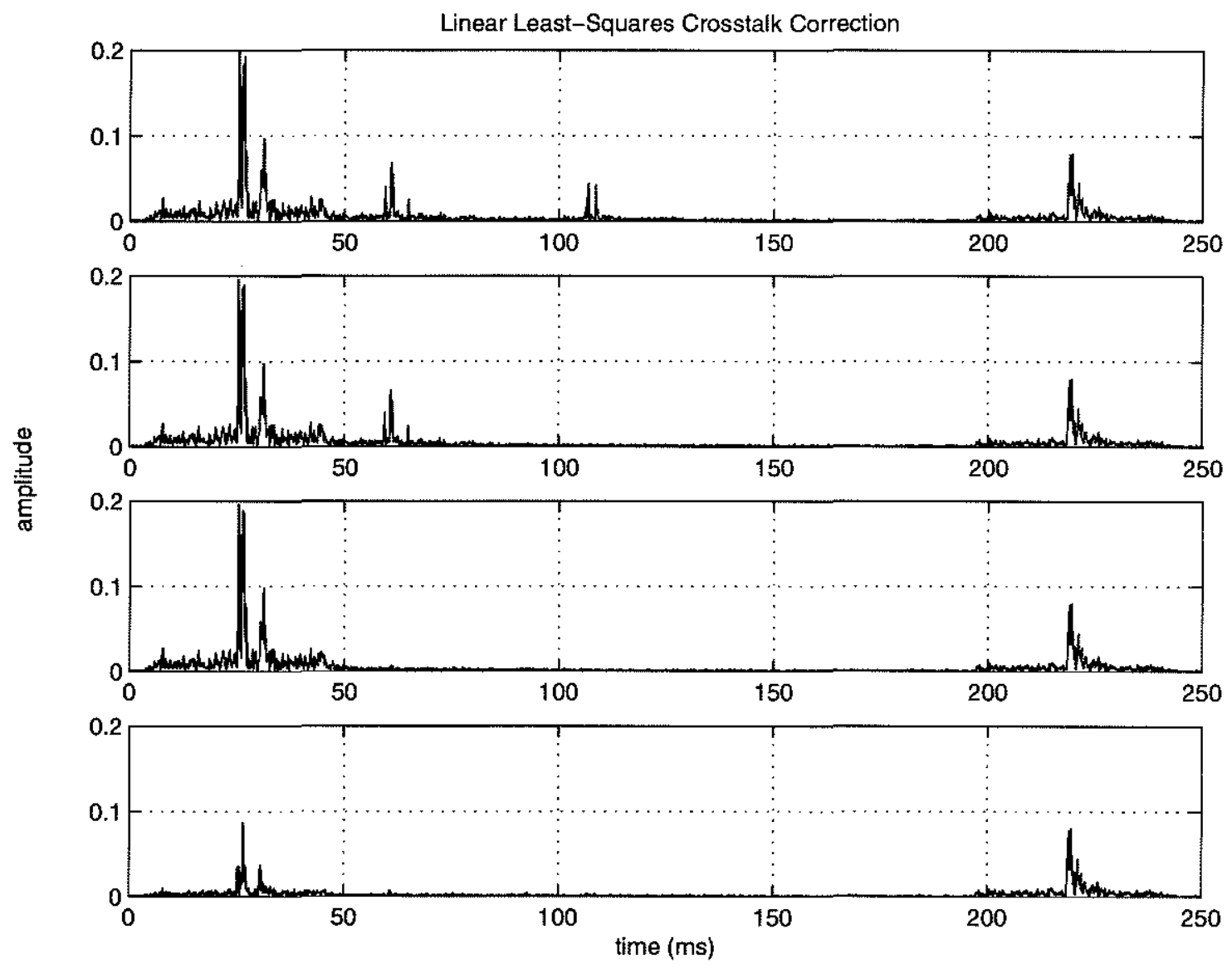

Figure 2-5: Frequency-independent crosstalk correction of inshore channel 1 . The uppermost plot shows the uncorrected, channel 1 signal as it was recorded. Moving down, the next plots correct for crosstalk from channel 10 , then channel 11, then channels 12-15. The large residual remaining in the lowest plot is due to the contributions of channel 16, which was not recorded. 
to crosstalk from the outer vertical array (hydrophones 12-16) can be removed as well. There is a relatively large energy residual from channel 16 , however, which was not recorded, and therefore cannot be properly removed.

Figure 2-6 shows the residual energy level as a function of time after crosstalk correction on channel 1. The acoustically-received energy on channel 1 is also plotted as a baseline for comparison. The residual energy from channel 10 crosstalk is small, almost always below the acousticallyreceived energy for channel 1, even when there is heavy acoustic attenuation. The residual energy from channel 11 crosstalk is quite similar to that from channel 10, although it is not as consistently below the acoustically-received energy levels for channel 1. Residuals from the outer vertical array, channels 12-16, are significantly larger, almost always larger than the acoustically-received energy on channel 1. This is again because channel 16 was not recorded, and so there is always a component of the crosstalk from the outer vertical array which cannot be cancelled with this technique.

\section{Time variability of crosstalk coefficients}

The energy residuals from the previous section were obtained by estimating the crosstalk coefficient for every impulse response estimate. In order to correct sections of data where transmission was continuous, and range-gating is not possible, the crosstalk coefficients must be stable enough to allow correction without continually tracking the coefficient estimates.

In Figure 2-7, the energy residuals are plotted as a function of time, both when estimating the coefficients separately at every impulse response estimate, and when applying an initial estimate to all later times. Crosstalk coefficient variability was examined on short timescales only, up to timescales of hours, but not days.

For channels 10 and 11, there is no significant difference in correction performance between tracking the coefficients, and using an initial estimate for subsequent data.

For channels 12-15, the energy residual is significantly larger when the crosstalk coefficients are not being continuously tracked. This is again due to channel 16 not being recorded, yet contributing heavily to the crosstalk. 

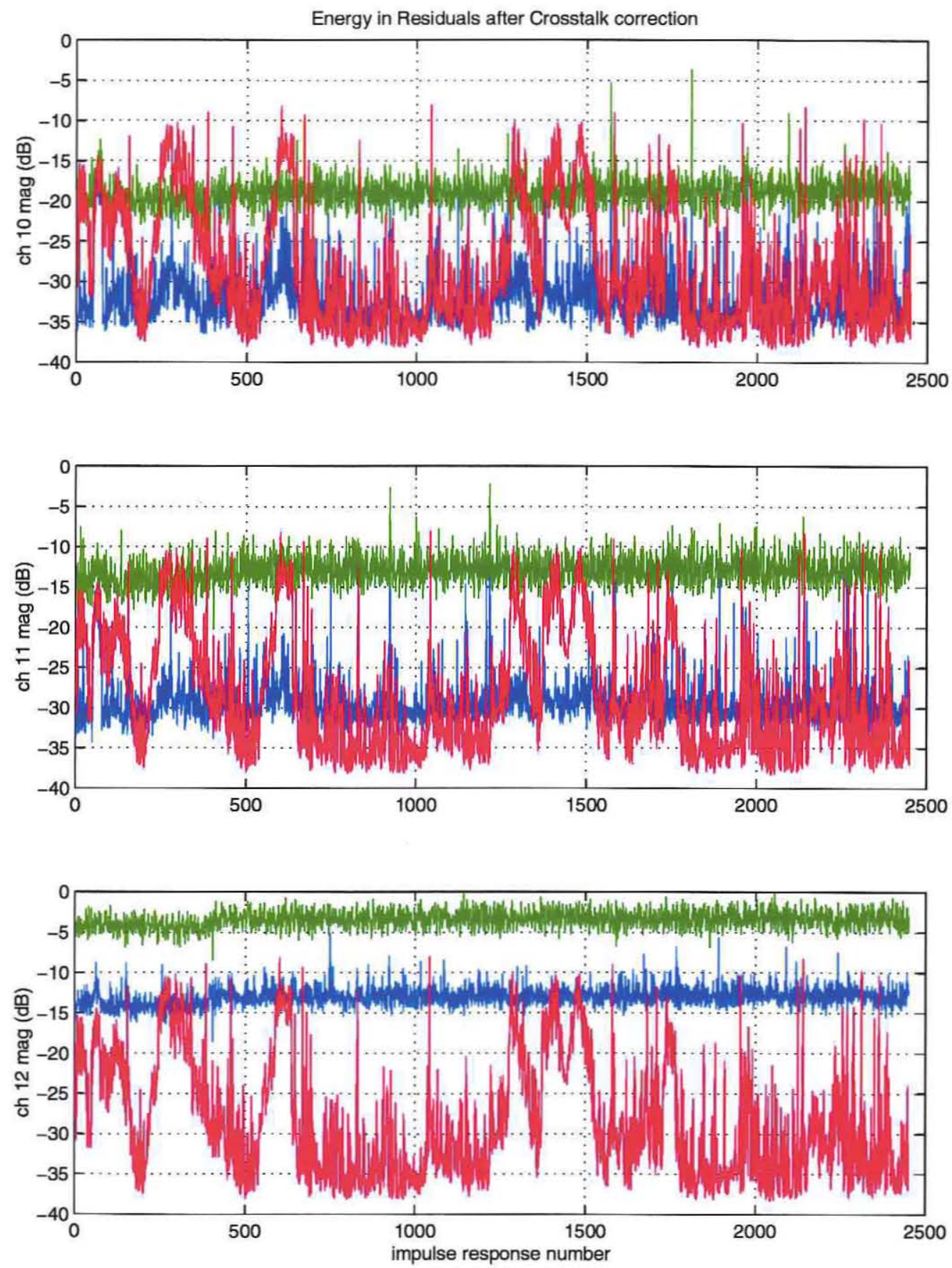

Figure 2-6: In each plot, the red curve, repeated identically in all three plots, shows the acousticallyreceived energy in channel 1 , for comparison. The green curves shows the crosstalk energy in channel 1 from channels 10,11 , and 12, for the top, middle and bottom plots, respectively. The blue curves show the residual crosstalk energy in channel 1, after crosstalk correction, due to crosstalk from channels 10,11 , and 12 , for the top, middle, and bottom plots, respectively. 

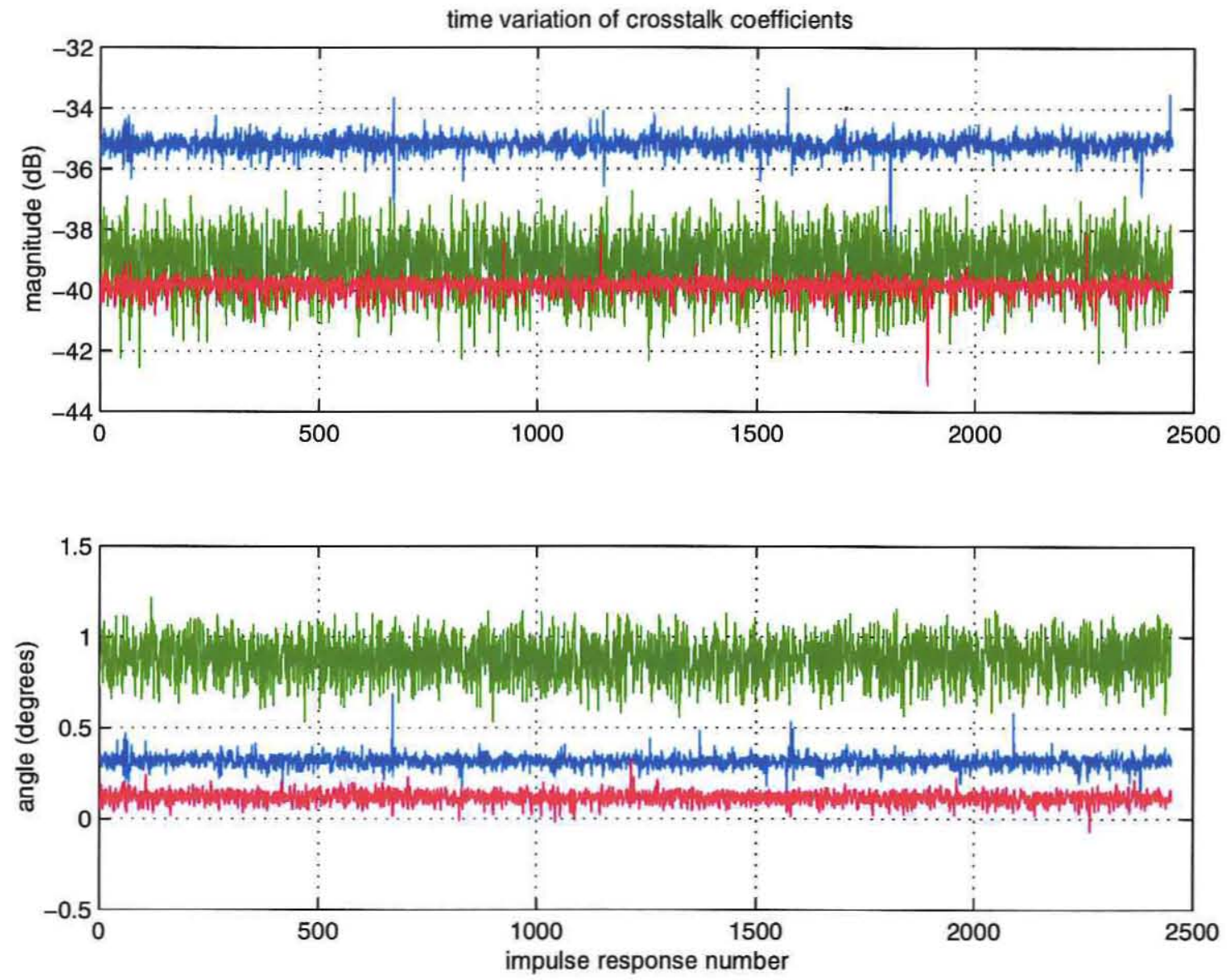

Figure 2-7: The upper and lower plots show the time variability of the magnitude and phase, respectively, of the single-tap coefficients for channels 10, 11, and 12. Channel 10 is plotted in blue, channel 11 in red, and channel 12 in green. Channel 12 has a significantly larger variance in both magnitude and phase, due to the contribution from the uncorrected channel 16. 


\subsection{Alternative approaches}

The fundamental problem with the crosstalk correction is that channel 16 was not recorded, and hence it is very difficult to correct for its effects. In theory, it might be possible to estimate channel 16 from channels $12-15$, since the hydrophones for those channels were in a five-element vertical array.

Using array processing to beamform the signals on channels 12-15, and then estimate the signal on channel 16, is not likely to work well, however. The acoustic wavelength in a band around $11.5 \mathrm{kHz}$ is about $13 \mathrm{~cm}$, but the vertical array spacing was $1 \mathrm{~m}$. The unambiguous range in angle before aliasing is therefore about $\pm 6^{\circ}$, but it is quite possible for high-angle reflections from the surface or bottom to arrive at the array.

Additional attempts to estimate channel 16 included an ad-hoc method to estimate the delay and phase of channel 16 with respect to channel 15 . Using a delayed and phase-shifted version of channel 15 to approximate channel 16 was not very successful, however, again most likely due to the relatively large separation of the sensors, reducing their correlation.

Further reading was done in noise cancellation and system identification techniques, without many promising leads[23, 7]. The signal on channel 16 is qualitatively similar to those on channels $12-15$, but the spatial separation is relatively large, and not precisely known, reducing the likelihood of making a good estimate of channel 16 based upon the received signals on channels 12-15. Without a good estimate of channel 16 , crosstalk correction cannot be performed effectively on signals which are not range-gated.

\subsection{Conclusions}

The coupling between the channels can be well modeled as a high-pass filter, leading to crosstalk. This effective high-pass filter, however, has a roll-off frequency which is much larger than the sampling frequency of the system. Because of this, estimates of the tap coefficients of any discrete-time frequency-dependent filter which approximates the high-pass filter will not be robust in even small amounts of noise. Practical crosstalk models for this system are therefore limited to single-tap, or frequency-independent, models.

Estimating the crosstalk coefficients from matched-filtered data, instead of the unfiltered baseband 
signals, has several advantages, most significantly that the variance of the estimates is lower. With single-parameter linear least-squares estimates applied to impulse response estimates, the crosstalk energy can be greatly reduced for some channels, in particular the mid-water hydrophones 10 and 11, when transmitting from deep-water source 4. For the deep-water vertical array, however, comprised of hydrophones 12-16, the residual crosstalk energy is significantly higher, since hydrophone 16 was powered but was not recorded, and cannot be corrected for using this technique. Furthermore, the energy residuals for channels $12-16$ are significantly increased when the coeffcients are not continuously tracked. This means that for signals which cannot be range-gated, for instance repeated maximal-length pseudo-random sequences, the crosstalk from the outer vertical array cannot be easily removed. In addition, channel 16 cannot be well-estimated from channels 12-15, either through array processing or other techniques, and so the large and time-varying energy residuals due to channel 16 cannot be eliminated.

The large residuals from channel 16 are only a problem when transmitting from the deep-water sources, however: for sources which were more distant from hydrophone 16, the crosstalk is not dominated by contributions from the deep-water vertical array. There is little high-quality data from the in-shore source, however, and signals from the mid-water source are difficult to range-gate for coefficient estimation. Another possibility is to use signals from the IOS sonar, which was near the in-shore source and transmitted throughout the experiment. The majority of the codes used by the IOS sonar cannot produce impulse response estimates, however, and even for the few true pseudo-random codes which were transmitted, the processing gain is relatively low, even compared with a length- 88 Turyn code. 


\begin{tabular}{|l||c|c|c|c|c|c|c|c|c|c|c|c|c|c|c|c|c|}
\hline \multicolumn{10}{|c|}{ Table 2-2: Measured Channel-to-Channel Shield Capacitance, nF (Datasonics) } \\
\hline \hline Channel & 1 & 2 & 3 & 4 & 5 & 6 & 7 & 8 & 9 & 10 & 11 & 12 & 13 & 14 & 15 & 16 \\
\hline 1 & & 0.168 & 0.182 & 0.197 & 0.198 & 0.168 & 38.00 & 33.00 & 0.19 & 52.00 & 44.20 & 46.00 & 35.50 & 54.00 & 36.10 & 34.80 \\
2 & 0.160 & & 0.165 & 0.195 & 0.198 & 0.166 & 0.183 & 0.185 & 0.181 & 0.195 & 49.10 & 43.20 & 37.30 & 55.00 & 56.00 & 36.00 \\
3 & 0.187 & 0.169 & & 0.197 & 0.192 & 0.184 & 0.171 & 0.192 & 0.174 & 0.193 & 0.20 & 37.00 & 35.00 & 49.00 & 47.00 & 32.00 \\
4 & 0.196 & 0.193 & 0.197 & & 0.185 & 0.197 & 52.00 & 0.17 & 49.00 & 0.186 & 44.30 & 36.00 & 47.00 & 36.00 & 37.00 & 35.20 \\
5 & 52.30 & 57.50 & 60.90 & 67.60 & & 59.70 & 57.80 & 66.90 & 50.80 & 82.00 & 51.50 & 32.20 & 34.50 & 36.60 & 42.30 & 30.30 \\
6 & 81.20 & 99.50 & 69.60 & 69.20 & 59.70 & & 58.30 & 91.40 & 58.00 & 61.20 & 45.00 & 35.40 & 41.90 & 44.60 & 42.30 & 30.20 \\
7 & 58.90 & 68.20 & 91.90 & 51.20 & 57.80 & 58.30 & & 54.50 & 83.40 & 55.00 & 64.80 & 32.80 & 31.60 & 43.10 & 42.10 & 29.30 \\
8 & 60.60 & 67.70 & 61.20 & 91.20 & 66.90 & 91.40 & 54.50 & & 51.30 & 76.00 & 44.90 & 37.30 & 44.80 & 39.00 & 38.30 & 36.40 \\
9 & 63.30 & 72.00 & 85.60 & 48.10 & 50.80 & 58.00 & 83.40 & 51.30 & & 49.00 & 48.00 & 33.20 & 30.90 & 51.60 & 37.10 & 29.00 \\
10 & 51.60 & 57.20 & 59.60 & 67.00 & 82.00 & 61.20 & 55.00 & 76.00 & 49.00 & & 47.60 & 32.20 & 35.10 & 36.00 & 45.00 & 30.40 \\
11 & 43.50 & 48.10 & 56.00 & 42.90 & 51.50 & 45.00 & 64.80 & 44.80 & 48.00 & 47.60 & & 28.10 & 25.80 & 33.40 & 44.90 & 30.40 \\
12 & 45.70 & 41.80 & 35.60 & 34.6 & 32.20 & 45.40 & 32.80 & 37.30 & 33.20 & 32.30 & 28.10 & & 28.80 & 29.60 & 24.70 & 36.50 \\
13 & 34.90 & 36.30 & 33.50 & 45.90 & 34.50 & 42.00 & 31.60 & 44.90 & 30.90 & 35.10 & 25.80 & 25.80 & & 26.40 & 25.00 & 37.40 \\
14 & 55.00 & 55.30 & 48.80 & 36.40 & 36.66 & 44.60 & 43.20 & 38.80 & 51.50 & 36.00 & 33.40 & 29.50 & 26.40 & & 28.30 & 24.80 \\
15 & 36.10 & 56.00 & 47.00 & 37.00 & 42.30 & 42.30 & 42.10 & 38.30 & 37.10 & 45.00 & 44.90 & 24.70 & 25.00 & 28.30 & - \\
16 & 34.80 & 36.00 & 32.00 & 35.20 & 30.30 & 30.20 & 29.30 & 36.40 & 29.00 & 30.40 & 30.40 & 36.50 & 37.40 & 24.80 & - & - \\
\hline
\end{tabular}




\section{Chapter 3}

\section{Acoustic Propagation Modeling in Bubble Clouds}

\subsection{Introduction and Motivation}

In the surf zone, breaking waves inject large amounts of air into the water. Turbulent flow mixes the resulting bubble clouds downwards into the water column, until they gradually rise to the surface or dissolve. The presence of the bubbles significantly affects acoustic propagation in the water, primarily by changing the water-air mixture's effective compressibility. By increasing the medium's compressibility, the bubbles lead to dramatically increased acoustic attenuation, and significantly reduced sound speeds, compared with non-bubbly water.

In heavy surf conditions, the breaking waves are often separated by less than the time required for the bubbles to rise to the surface or to dissolve. In this situation, continued injections of bubbles from new breaking waves lead to the continuous presence of bubble clouds. Due to the heavy acoustic attenuation caused by bubbles, this can lead to extended periods where effectively no sound can travel in the region of the bubble clouds. This is a challenge for underwater acoustic communication in the surf zone.

Being able to model acoustic propagation in the presence of bubble clouds is therefore important in predicting the performance of underwater acoustic communication systems for the surf zone. The Scripps Pier experiment in May 1999 provided a good opportunity to compare modeled propagation with measurements of the acoustic communication channel. The conditions during 
the Scripps Pier experiment provided a difficult communication channel: bubble-induced attenuation was as high as $26 \mathrm{~dB} / \mathrm{m}$, causing total channel outages, which could last for several minutes[6].

With potentially limited opportunities for communication, an acoustic communication system for the surf zone should transmit as soon as possible after the channel clears. In the Scripps Pier experiment, several instances of channel clearing were both observed, then modeled. The received signal energy from an acoustic probe was measured over time, and required close to $50 \mathrm{~s}$ to recover to half its original energy from a $50 \mathrm{~dB}$ fade, caused by a bubble injection from a breaking wave. Modeling this same event, using a geometric ray model of acoustic propagation, predicted that the signal energy would have recovered after only 20 seconds[6].

One possible explanation for this discrepancy is that another breaking event, smaller and hence unnoticed in the observations, injected additional bubbles into the water, delaying the signal recovery. Another possible explanation is that turbulent flow increases the buoyant rising time of bubbles. A third possible explanation, investigated in this chapter, is that a simple geometric ray model is not sufficient for modeling the signal recovery after a channel outage. It may be necessary to consider the attenuation characteristics of the entire water column when modeling channel outages, rather than simply considering the attenuation along each ray path.

The hypothesis tested in this chapter is that attenuation in excess of that predicted by a geometric ray model can occur during times when a bubble cloud partially obscures the acoustic channel. This chapter will present both the modeling approach and results. A full wave propagation model is used in order to minimize bias caused by a priori modeling assumptions. The simulations attempt to reproduce the main features of the Scripps Pier environment, namely the up-wedge propagation and the bubble clouds near the inshore hydrophones. The results are investigated in terms of channel impulse responses and their angles of arrival, to determine if the full wave propagation needs to be taken into account to model surf zone propagation in the presence of bubble clouds.

\subsection{Modeling Approach}

\section{Oases}

The acoustic propagation package Oases was used to model propagation in the environment both with and without bubble clouds, to test whether a bubble cloud near the surface will prevent 
significant propagation under the cloud. Oases performs direct integration of the wave equation, without assumptions such as the applicability of mode or ray models.

Oases calculates the exact wave equation solution in a finite element cell, combining the solutions from adjacent cells by matching boundary conditions. Finite element cells are stacked vertically into horizontally-stratified range-independent environments. Within each range-independent stack of cells, the wave equation can be separated (or, equivalently, transformed) into a depth-dependent wave equation. The solution to the depth-dependent wave equation is a depth-dependent Green's function. Performing a Hankel transform, or wavenumber integration, between cylindrical (constantdepth) wavenumber and cylindrical range yields a pressure field transfer function as a function of range and depth. From this point, an inverse Fourier transform, or frequency integration, can be used to yield a time-domain impulse response as a function of range and depth[17, 11].

Within each cell, the sound speed, attenuation, and density of the medium has to be specified. Environments which are not range-independent can be approximated by concatenating short, finely stepped range-independent environments.

\section{Modeled Environment}

To test whether a geometric ray model is sufficient for modeling acoustic propagation under surfzone bubble clouds, an Oases model was constructed of the Scripps Pier environment. The acoustic propagation path was modeled as a wedge, as shown in Figure 3-1. In the experiment, the source was $1 \mathrm{~m}$ off the bottom, in $7 \mathrm{~m}$ of water, and propagation was up-slope to an inshore array of receivers in $1.5 \mathrm{~m}$ of water, about $300 \mathrm{~m}$ away. The bottom slope is therefore $1.8 \%$. The water column was modeled as fully-mixed, with a constant speed of sound and density, $1500 \mathrm{~m} / \mathrm{s}$ and $1 \mathrm{~g} / \mathrm{cm}^{3}$, respectively. The acoustic attenuation in the water column was about $0.0001 \mathrm{~dB}$ per wavelength near the center frequency of $11.5 \mathrm{kHz}$, with a slight frequency dependence. Similarly, the sand bottom was modeled as uniform, with a constant sound speed, density, and acoustic attenuation of $1700 \mathrm{~m} / \mathrm{s}, 1.5 \mathrm{~g} / \mathrm{cm}^{3}$, and $0.5 \mathrm{~dB}$ per wavelength, respectively[11, 3]. The half-space above the water's surface was modeled as a vacuum.

The bubble cloud was modeled as a rectangular region with a depth-dependent void fraction and a single fixed bubble size of $100 \mu \mathrm{m}$. The void fraction varied linearly (in several discrete steps) from $10^{-6}$ at the bottom of the cloud to $10^{-4}$ at the surface. This models a partially evolved cloud, 


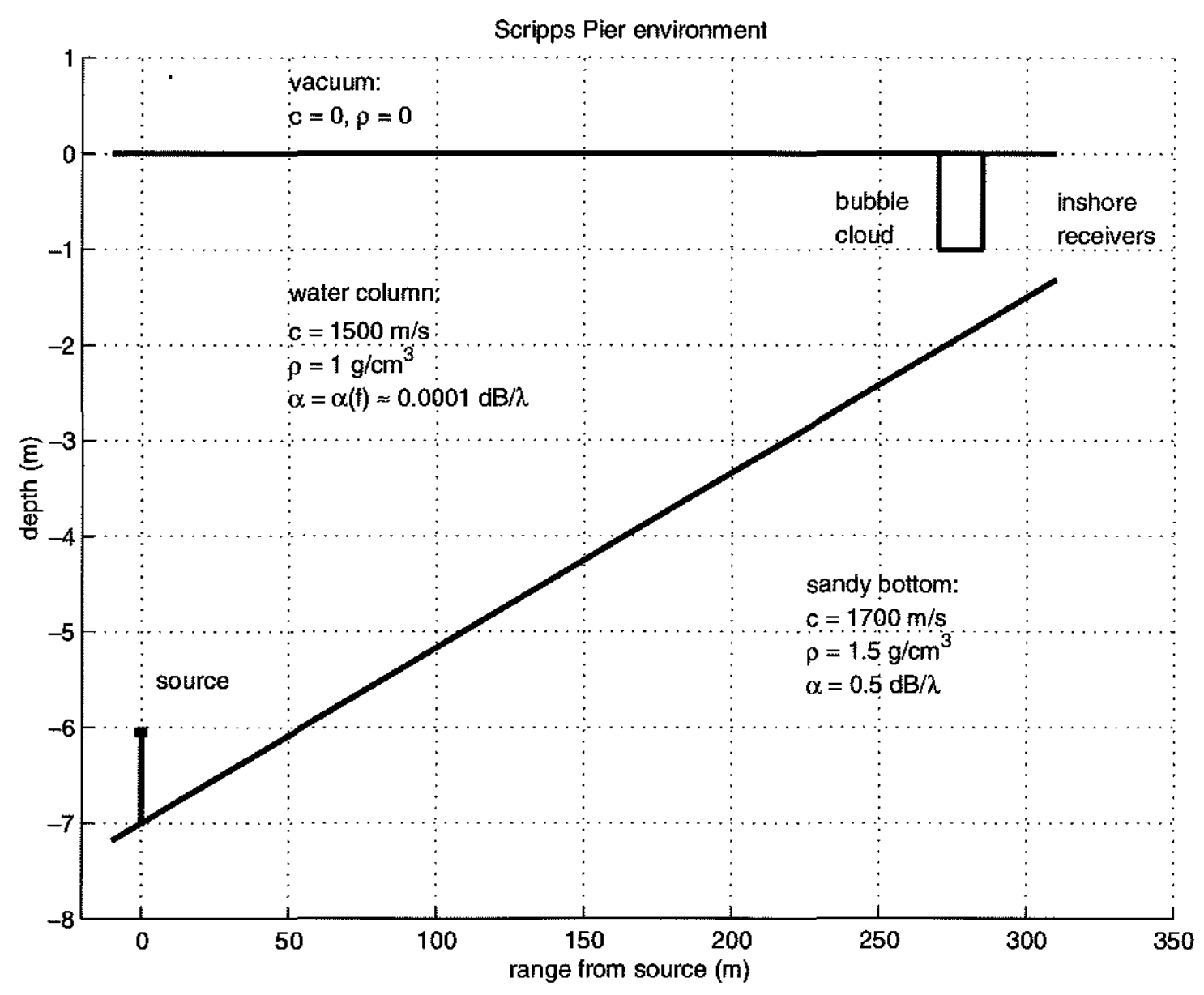

Figure 3-1: Model surf zone environment. 
with bubbles having risen to create a larger void fraction near the surface. While realistic bubble clouds may have a different depth-dependent void fraction, the modeled void fraction is sufficient to test the hypothesis under investigation. The core of the bubble cloud had an extent of $15 \mathrm{~m}$ in range, with tapered void fractions both seaward and shoreward of the core cloud, so there were no sharp, sudden changes in sound speed due to a discontinuity in void fraction. The depth of the cloud was varied between runs. In some runs, there was no cloud; in others, the cloud depth was $0.5 \mathrm{~m}, 1.0 \mathrm{~m}$, or $1.5 \mathrm{~m}$. Within the cloud, the sound speed and attenuation were calculated using Commander and Prosperetti's formulas, as described in the next section.

\section{Sound Speed and Attenuation in Bubbly Water}

The air in bubbles is far more compressible than the surrounding water, which leads to a large effect on sound propagation, both in the effective speed of sound and in its attenuation.

As an intuitive, physically-based explanation, the speed of sound in a medium is given by its density, $\rho$, and its elasticity, or bulk modulus, $E$ :

$$
c=\sqrt{\frac{E}{\rho}} .
$$

In a bubbly water mixture with a void fraction $\beta$, the effective density is given by

$$
\rho_{\text {eff }}=\beta \rho_{\text {air }}+(1-\beta) \rho_{\text {water }} .
$$

The effective density is approximately equal to the density of water, since typically, the void fraction is very small, and the density of air is negligible compared to that of water. The mixture's effective bulk modulus is

$$
\frac{1}{E_{\text {eff }}}=\frac{\beta}{E_{\text {air }}}+\frac{1-\beta}{E_{\text {water }}}
$$

The bulk modulus of air is much less than that of water, so, due to the inverse relationship, the low bulk modulus of air contributes heavily to the effective bulk modulus of the mixture, despite typically small void fractions. Due to the large decrease in effective bulk modulus in bubbly water, the average sound speed can be quite low, down to $800 \mathrm{~m} / \mathrm{s}$ or lower, compared with around $1500 \mathrm{~m} / \mathrm{s}$ for non-bubbly water under typical conditions.

Air bubbles also considerably increase the attenuation of water. Without bubbles, salt water at- 
(a) acoustic attenuation in salt water.

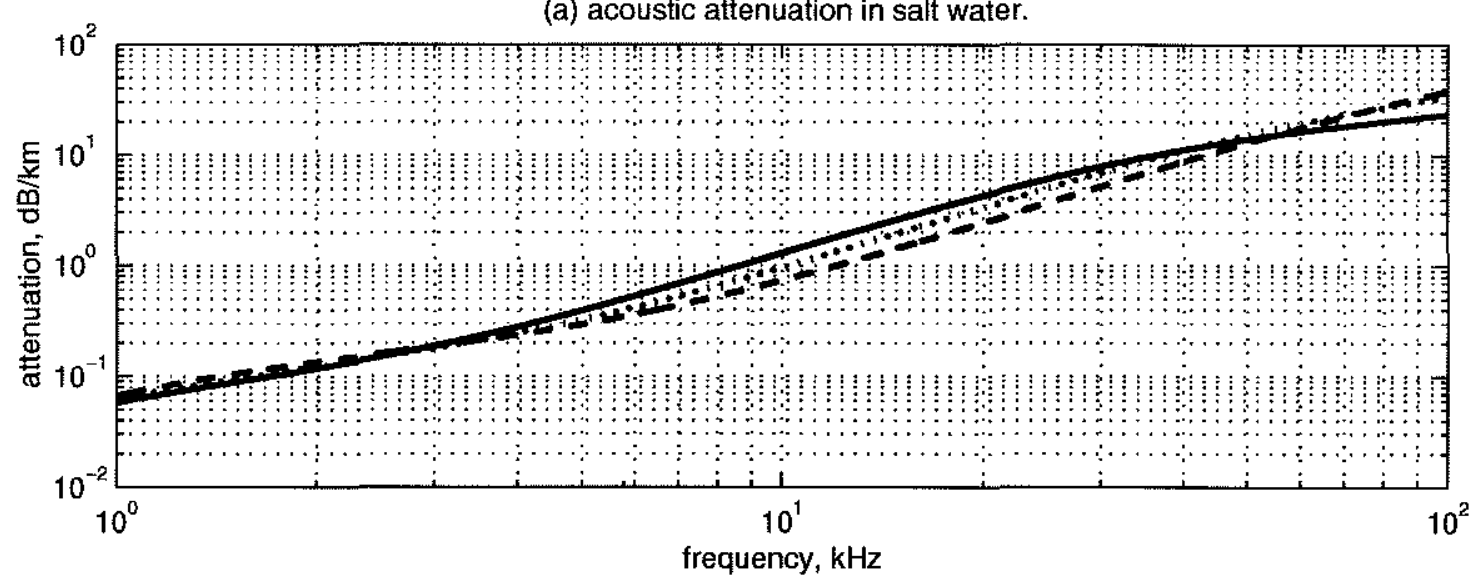

(b) resonant frequencies of bubbles in water.

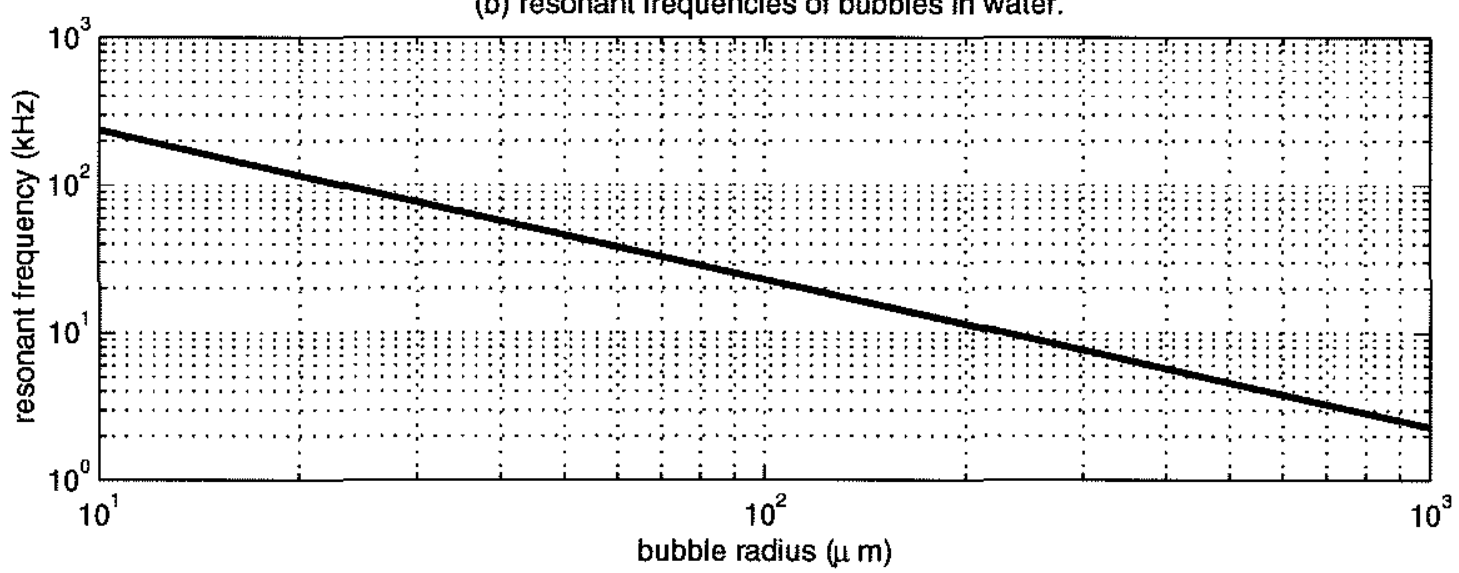

Figure 3-2: (a) Attenuation of non-bubbly water, at $0^{\circ} \mathrm{C}$ (solid line), $10^{\circ} \mathrm{C}$ (dotted line), and $20^{\circ} \mathrm{C}$ (dashed line). (b) Resonant frequency of bubbles as a function of bubble radius.

tenuates sound due to water's natural viscosity, and also due to resonances in the various salts. This attenuation is shown in Figure 3-2a. Near $10 \mathrm{kHz}$, and in typical conditions, the attenuation of an acoustic signal is about $0.0001 \mathrm{~dB}$ per wavelength[13].

In a bubbly mixture, the bubbles will have a resonant frequency for mechanical oscillations. Driving the bubbles acoustically near their resonant frequency will lead to acoustic attenuation, primarily due to scattering from the bubbles. Additional attenuation is due to the thermal conductivity within the bubble and the shear viscosity at the surface of the bubble. The resonant frequency of a bubble of radius $a$, with ambient water pressure $P$ and air density $\rho_{\text {air }}$ is

$$
f_{R}=\frac{1}{2 \pi a} \sqrt{\frac{3 \gamma P}{\rho_{\mathrm{air}}}} g(\cdots),
$$


where $\gamma$ is the ratio of specific heats for air, and $g(\cdots)$ is a correction due to the bubble's surface tension and non-adiabatic oscillation. Near the surface, a $100 \mu \mathrm{m}$ bubble - typically the peak of the surf-zone bubble size distribution - has a resonant frequency of $33 \mathrm{kHz}$, as can be seen in Figure $3-2 \mathrm{~b}[3]$.

A more accurate, and more involved, derivation of the sound speed and attenuation in a bubbly water mixture is developed by Commander and Prosperetti. Away from bubble resonances, and for relatively small void fractions (below about $2 \%$ ), their formulas yield sound speed and attenuation as functions of depth, void fraction, bubble radius, source frequency, and the nominal sound speed. Their derivation combines a wave equation for an averaged bubbly mixture with thermodynamic relations for a bubble, linearizing to give the complex sound speed in the mixture.

The expression for complex sound speed is a function of the bubble radius distribution, $f(a)$, where $a$ is the bubble radius, as well as driving frequency $\omega$, nominal sound speed $c$, and bubble parameters $\omega_{o}$ and $b$.

For a bubble distribution with a single bubble radius $a_{o}$, the bubble radius distribution function becomes

$$
f(a)=n \delta\left(a-a_{o}\right)=\frac{\beta}{\frac{4}{3} \pi a_{o}^{3}} \delta\left(a-a_{o}\right),
$$

where $n$ is the number of bubbles per unit volume. The parameters $\omega_{o}$ and $b$ are analogous to the resonant frequency and the damping coefficient of a linear oscillator. The analogy serves primarily for intuition, and cannot be fully exploited, since these parameters depend upon driving frequency, which is not the case for a linear oscillator. The damping term, $b$, arises from viscous, thermal, and, acoustic scattering effects, while the analogous term to resonant frequency, $\omega_{0}$, is due to both mechanical resonances, and thermal effects in both the water and air.

Commander and Prosperetti $[4,15]$ express the complex sound speed in the mixture of water and air, $c_{m}$, in a ratio with the nominal sound speed, $c$ :

$$
\frac{c^{2}}{c_{m}^{2}}=1+4 \pi \omega^{2} \int_{0}^{\infty} \frac{a f(a) d a}{\omega_{o}^{2}-\omega^{2}+2 i b \omega} .
$$

For distributions with a single bubble radius, $f(a)$ is a delta function, and the expression becomes

$$
\frac{c^{2}}{c_{m}^{2}}=1+\frac{3 c^{2} \beta}{a_{o}^{3}} \frac{1}{\omega_{o}^{2}-\omega^{2}+2 i b \omega} .
$$


To determine the acoustic phase speed and attenuation in the bubbly mixture, consider a propagating complex exponential wave, $\exp \left(i \omega t-i k_{m} x\right)$, where $k_{m}$ is the complex wavenumber in the mixture. Letting $k_{m}=\frac{\omega}{c}(u-i v)$, the expression for the propagating wave then becomes

$$
\exp \left(i \omega t-i k_{m} x\right)=\exp \left(\operatorname{Im}\left(k_{m}\right) x\right) \exp \left(i \omega\left(t-\frac{\operatorname{Re}\left(k_{m}\right)}{\omega} x\right)\right)=\exp (-A x) \exp \left(i \omega\left(t-\frac{x}{V}\right)\right)
$$

The expression farthest to the right describes a general propagating wave with an attenuation per unit length of $A$ and a phase speed $V$. Identifying terms, the phase speed and attenuation are related to the real and imaginary parts of the wavenumber, respectively. The attenuation per unit length is then $A=-\operatorname{Im}\left(k_{m}\right)=\omega v / c$; in decibels per unit length, it is $A_{\mathrm{dB}}=20\left(\log _{10} e\right)(\omega v / c)$. The phase speed is $V=\omega / \operatorname{Re}\left(k_{m}\right)=c / u$.

Experimental data compares well with sound speeds and attenuations derived from these equations[4].

\section{Stepped Wedge and Model Convergence}

Once the sound speed and attenuation can be calculated within each of the range cells, the next step in creating the modeled environment is to model the bathymetry at Scripps Pier. The geometry at Scripps Pier can be closely approximated by a wedge, with the source at the deep end, and the receiver arrays upslope from there. Environments with range-varying bathymetry, or rangedependent environments, cannot be modeled directly in Oases, as it makes use of exact solutions to the wave equation in range-independent finite element cells. A range-dependent environment for Oases, such as a wedge, can however be constructed by connecting short range-independent environments.

In creating a range-dependent wedge environment from many discrete steps of varying depth, determining the number of steps to use is the central question. Too few steps will give a poor approximation to a smooth wedge, while too many steps will increase the computational cost, and could actually reduce accuracy due to accumulation of numerical roundoff errors.

Acoustic propagation up a smooth wedge leads to standard specular reflections from the wedge surface. Plane waves will be reflected as plane waves, as shown in Figure 3-3a. The reflection of a plane wave from a stepped wedge, however, will yield a ragged wavefront, as in Figure 3-3b. When the ragged wavefront deviates significantly from a plane wavefront, then the step size is too coarse 
(a) Forward scatter from a smooth wedge

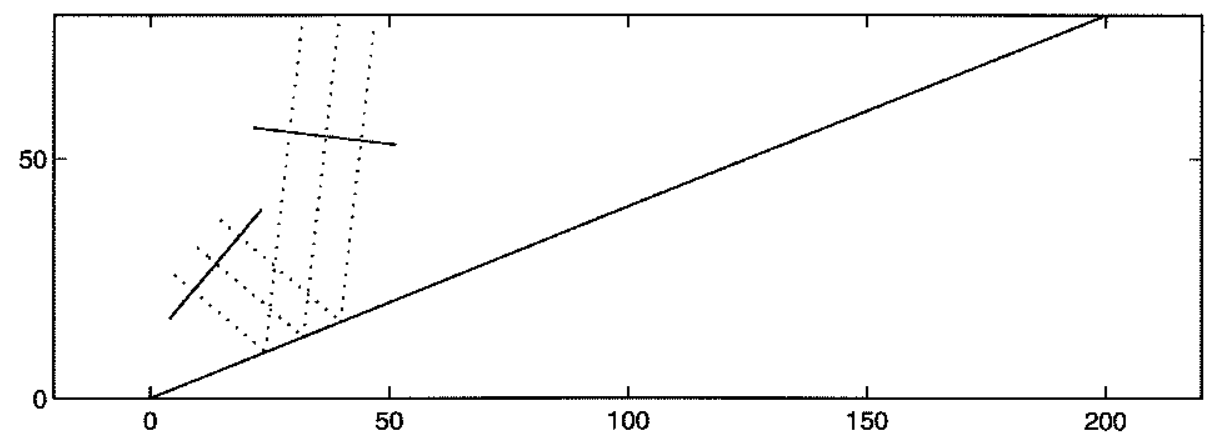

(b) Forward scatter from a stepped wedge

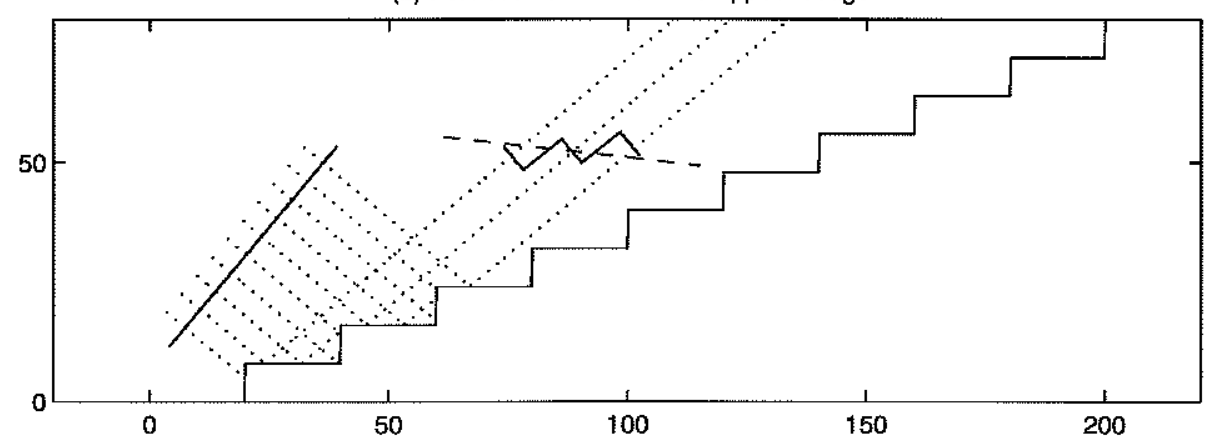

Figure 3-3: Reflection from a smooth wedge (a) and a stepped wedge (b). The rays are plotted with dotted lines, with wavefronts in solid lines. For the reflection from a stepped wedge, the smoothwedge reflection wavefront is plotted with a dashed line in addition to the ragged wavefront. 
for a good simulation result. In terms of horizontal step size, this criterion becomes

$$
\Delta x \ll \frac{\lambda}{2 \sin \phi \tan \theta}
$$

where $\Delta x$ is the horizontal width of the step, $\lambda$ is the acoustic wavelength, $\phi$ is the angle of incidence, and $\theta$ is the angle of the wedge, both with respect to the horizontal[10].

Ideally, to test this criterion for convergence, the analytic result would be compared to numerical runs at increasing numbers of steps. When the error between analytic and numerical solutions became sufficiently small, that would be the number of bathymetry steps to use to approximate the wedge. An analytic solution only exists for a "hard" bottom, however, in which the sound speed in the the bottom is infinite. Furthermore, even for the hard-bottom case, the analytic solution involves an infinite sum of integrated high-order Bessel functions, which would be very susceptible to numerical round-off error, reducing its usefulness as a baseline result[9].

Instead, a convergence test can take a run with a very large number of bathymetry steps, and assume that in this case the solution is fully converged. Plotting the energy in the difference between this case and runs with fewer steps should show a point beyond which additional steps have a negligible improvement in energy residual. This can be taken to be the appropriate number of bathymetry steps for the simulation.

To calculate the residual energy in the difference between the run at a very large number of steps ( $N$ steps) and intermediate runs of $n$ steps, two metrics were used. Both metrics compared a small rectangle within the range-depth plot of the complex pressure field, for both $N$ steps and $n$ steps. Let $P_{n}$ be a rectangular matrix of pressures, with the columns incrementing in range and the rows incrementing in depth, calculated by Oases using an environment with $n$ bathymetric steps.

The first metric was

$$
m_{1}(n ; N)=10 \log _{10}\left\{\frac{\sum_{i, j}\left|P_{n}-P_{N}\right|^{2}}{\sum_{i, j}\left|P_{N}\right|^{2}}\right\},
$$

where the sums over $i$ and $j$ are sums over the rows and columns of the pressure field matrices. This metric is just the element-by-element residual energy, normalized by the total energy in the run with $N$ bathymetric steps, which was assumed to be fully converged. 

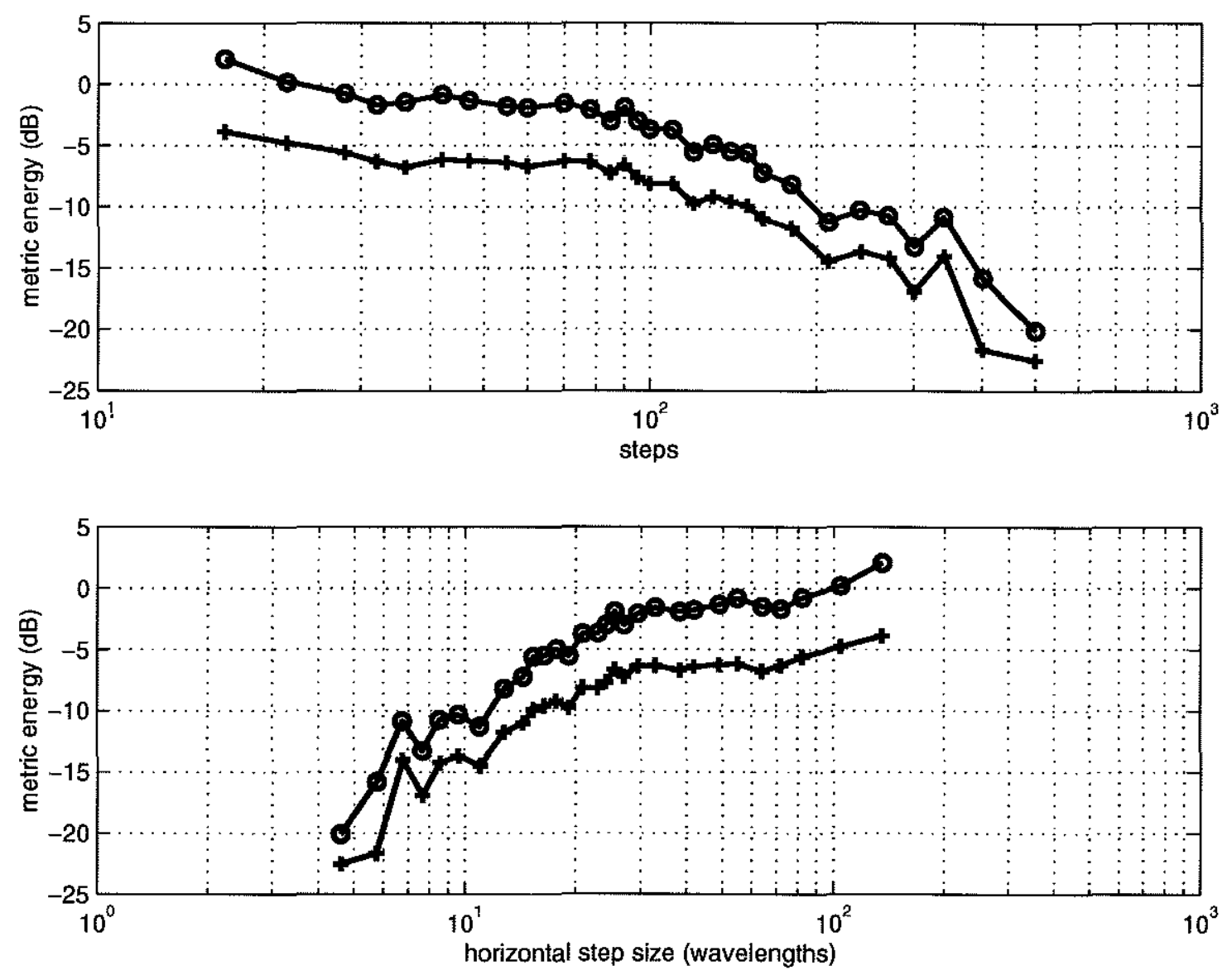

Figure 3-4: Convergence of pressure field in the bubble cloud region in a stepped wedge environment. Metric 1 is plotted with circles; metric 2 with crosses. Numerical round-off errors cause the residual energy metrics to increase beyond 600 steps, despite still having a horizontal step size of several wavelengths.

The second metric was quite similar:

$$
m_{2}(n ; N)=10 \log _{10}\left\{\frac{\sum_{i, j}\left(\left|P_{n}\right|-\left|P_{N}\right|\right)^{2}}{\sum_{i, j}\left|P_{N}\right|^{2}}\right\} .
$$

In this case, only the magnitudes of the pressure fields are considered; phase is ignored. By ignoring any possible errors in phase, this metric will always give a lower residual energy.

The convergence tests were run on a wedge as described above in the environmental modeling section: $300 \mathrm{~m}$ long, $7 \mathrm{~m}$ deep at the source, $1.5 \mathrm{~m}$ deep at the inshore receivers. The results of the residual energy metrics are plotted in Figure 3-4. The range-depth square used was a $101 \times 161$ region $(12.6 \mathrm{~m}$ by $2.8 \mathrm{~m})$ in a run without a bubble cloud, but in the location where the cloud 
would have been. The metrics are plotted versus number of steps and also horizontal step width in wavelengths.

At first, the residual energy decreases as the number of steps increases (and therefore as the step size decreases). When using more than about 600 horizontal steps, however, the energy residuals start to increase again. This is most likely due to accumulated numerical round-off errors, effectively limiting the maximum number of steps which can be used. There was no clear sign that the pressure field had fully converged, despite using up to 1000 steps over $300 \mathrm{~m}$ at a wavelength of $0.13 \mathrm{~m}$. This corresponds to a horizontal step width of $2.3 \lambda$. This is consistent with Jensen's results, where forward scatter problems largely converged with step widths of $1.67 \lambda$, but not significantly more.

To avoid the numerical problems for the final simulation runs, the environments were made shorter $(150 \mathrm{~m})$ and wider $(10 \lambda)$ steps were used. This allowed fewer steps $(100)$ and therefore less accumulation of round-off errors. Although the pressure field will not have fully converged with these coarse steps, visual inspection of the range-depth plots of the pressure field shows that in fact the convergence is quite good, at least when comparing the plots to others made with fewer than 600 bathymetry steps.

\section{Oases Input and Output Files}

Once the modeled environment is fully determined, with a sufficient number of bathymetry steps, and a bubble cloud with realistic values for sounds speed and attenuation, Oases needs to be run to model the propagation. Appropriate input files need to be generated, and the output files need to be interpreted and processed.

Oases input files describe the environment as well as the source and receivers. The environment is described in terms of depths of layers, and the sound speed, attenuation, and density within those layers. The determination of these parameters was described in the preceding sections. In addition to the physical positions of the source and receivers, the range of frequencies for which to calculate the transfer function must be specified; for the Scripps Pier experiment, this was a $5 \mathrm{kHz}$ band from $9 \mathrm{kHz}$ to $14 \mathrm{kHz}$. Finally, the sampling at the receivers must be described, in terms of the separation of samples in time and the number of samples to generate. 
The sample spacing, $\Delta t$, must satisfy Nyquist sampling at the highest frequency of interest. This sets

$$
\Delta t \leq \frac{1}{2 \cdot 14 \mathrm{kHz}}=35.7 \mu s
$$

In these simulations, $\Delta t$ was chosen to satisfy this Nyquist sampling condition with equality. The number of samples in the time domain also needs to be specified, in particular to avoid timedomain aliasing of the impulse responses. The longest observed impulse responses in the Scripps Pier experiment were approximately $25 \mathrm{~ms}$; the simulated environment should have similar channel spreads. With the previously set value for $\Delta t$, at least 700 time-domain samples will be required for a $25 \mathrm{~ms}$ channel spread. Oases, however, requires that the number of time-domain samples be a power of 2, to allow the use of fast Fourier transforms. Using 1024 time-domain samples, the maximum channel spread will be $36.6 \mathrm{~ms}$. The channel spread also sets the frequency spacing in Oases. In this case, the frequency spacing between points in the transfer function will be

$$
\Delta f=\frac{1}{1024 \cdot \Delta t}=27.3 \mathrm{~Hz}
$$

Output files from Oases consist of the transfer function, as a function of frequency, calculated for many ranges and depths. The source is normalized to unit pressure, so the transfer function at a particular point is just the complex pressure field as a function of frequency, $p\left(f_{k} ; z, r\right)$. In this expression, $z$ and $r$ are the depth and range of the point, respectively, and $f_{k}$ is a frequency index for the discrete transfer function. The range of frequencies for which the transfer functions are calculated is not in general centered on zero. The transfer functions for these particular simulations covered the $5 \mathrm{kHz}$ band from approximately $9 \mathrm{kHz}$ to approximately $14 \mathrm{kHz}$ with a spacing of $27.3 \mathrm{~Hz}$. The corresponding frequency indices for which the transfer functions were calculated were therefore 330 to 512 , giving precise frequencies of $9024.5 \mathrm{~Hz}$ to $14001.7 \mathrm{~Hz}$, spaced by $27.3 \mathrm{~Hz}$.

Using a discrete-time frequency index $k$, corresponding to frequency $f_{k}$, the transfer function $H$ at a fixed depth $z$ and range $r$ is then

$$
H[k ; z, r]=p\left(f_{k} ; z, r\right), \quad 330 \leq k \leq 512 .
$$




\section{Transmission Loss, Impulse Responses, and Angle of Arrival}

Having generated an Oases output file containing transfer function coefficients, the data must be processed and interpreted.

The most direct way to inspect the results is with a transmission loss plot, plotted as pressure field magnitude versus range and depth. These plots are generated at a single frequency only, and show where nodes and peaks exist in the diffraction patterns for monochromatic propagation. Regions which are shadowed acoustically, such as behind the bubble clouds, can also be identified, as can regions with heavy attenuation, for example within the clouds themselves.

The transmission loss information, however, is a narrow-band representation of the channel. It reflects transmission loss when the transmitted signal is a continuous complex exponential and does not yield information about path-specific attenuation by direct inspection.

The next approach to viewing the data is to transform the transfer function into the time domain, creating an impulse response. The impulse responses show both the delay spread of the channel and the attenuation of each arrival. It can be further transformed to yield information on the signal's angle of arrival.

To transform the transfer function into the time domain, an inverse discrete-time Fourier transform is applied. The transfer function in the Oases output files is not immediately ready to be transformed, however. The frequency indices in the transfer function range from 330 (near $9 \mathrm{kHz}$ ) to 512 (near $14 \mathrm{kHz}$ ), and are not centered around zero. The transfer function can be written in notation similar to Matlab as

$$
H[k ; z, r]=\left[\mathrm{H}_{330} \cdots \mathrm{H}_{512}\right] .
$$

Shifting the coefficients in the transfer function vector is equivalent to demodulating the signal to baseband in the time domain. The center coefficient is $H_{421}$, so the transfer function vector after demodulating to baseband is

$$
H_{\mathrm{bb}}[k ; z, r]=\left[\mathrm{H}_{421} \cdots \mathrm{H}_{512} ; \mathrm{H}_{330} \cdots \mathrm{H}_{420}\right] .
$$

The inverse discrete-time Fourier transform can be applied to the baseband transfer function, $H_{\mathrm{bb}}$. 
There are 183 frequency-domain samples in the transfer function vector, and therefore there will be 183 time-domain samples in the corresponding impulse response vector. As described above, the total length of the impulse response is $36.6 \mathrm{~ms}$, so each impulse response sample in time would be $0.2 \mathrm{~ms}$ long. Padding the transfer function vector with zeros in the high-frequency (middle) samples will change the time-domain sample length. The original time resolution, $35.7 \mu \mathrm{s}$, as calculated by Oases, was achieved with 1024 samples in the impulse response. Because the transfer function has only 183 samples, the time resolution cannot be improved beyond $0.2 \mathrm{~ms}$, but the original sampling density, with smearing, can be recovered by padding the transfer function to have length 1024:

$$
H_{\text {final }}[k ; z, r]=\left[\mathrm{H}_{421} \cdots \mathrm{H}_{512} ; \operatorname{zeros}(1024-183,1) ; \mathrm{H}_{330} \cdots \mathrm{H}_{420}\right]
$$

The complex baseband impulse response at depth $z$ and range $r$ is therefore the inverse discretetime Fourier transform of $H_{\text {final }}$ :

$$
h[n ; z, r]=\frac{1}{N} \sum_{k=0}^{N-1} H_{\text {final }}[k ; z, r] e^{2 \pi i k n / N}, \quad 0 \leq n \leq N-1,
$$

where $N=1024$ due to the zero-padding.

The maximum range of the modeled environment was $150 \mathrm{~m}$, reduced from the $300 \mathrm{~m}$ environment at Scripps pier to minimize effects of the accumulation of numerical roundoff errors. At the nominal sound speed of $1500 \mathrm{~m} / \mathrm{s}$, the travel time for an acoustic signal is $100 \mathrm{~ms}$, which is more than the maximum delay spread allowed by the simulation sampling, $36.6 \mathrm{~ms}$. The impulse responses therefore will be aliased about the time origin, and can be given arbitrary delay offsets. Within an individual impulse response, however, there will be no internal aliasing, so long as the assumption of a channel spread of less than $36.6 \mathrm{~ms}$ holds.

An additional way to process the transfer function data is to plot the angle of arrival as a function of arrival time.

Starting with the complex pressure field $p\left(f, z, r_{o}\right)$ at a fixed range $r_{o}$, the range can be suppressed in the notation. Using array processing concepts to beamform the pressure field yields the angle 
of arrival as a function of frequency:

$$
\tilde{p}(f, \theta)=\sum_{z} p(f, z) e^{-i k(f) z \sin (\theta)}
$$

where $k(f)=2 \pi f / c$ is the acoustic wavenumber, and the angle of arrival $\theta$ ranges between $\pm \theta_{\max }$, and is with respect to the horizontal.

With an inverse discrete-time Fourier transform to transform to the time domain, the angle of arrival as a function of frequency is

$$
\tilde{p}(t, \theta)=\operatorname{IDFT}\{\tilde{p}(f, \theta)\},
$$

where the pressure field $p(\tilde{f}, \theta)$ must again be shifted to demodulate the time-domain signal to a complex baseband signal.

\subsection{Results}

The Oases runs were made with a wedge environment similar in character to the Scripps Pier environment. In the actual simulation runs, the number of bathymetry steps was limited to 100 , in order to reduce the effects of numerical round-off accumulation. Because of the reduction in the number of steps, the range was also reduced, from $300 \mathrm{~m}$ as in the actual Scripps Pier environment, to $150 \mathrm{~m}$. The slope of the bottom was kept the same, at $1.8 \%$, and the final depth was again $1.5 \mathrm{~m}$. The source was $1 \mathrm{~m}$ off the bottom, at the deepest point of the wedge, $4.25 \mathrm{~m}$.

\section{Range-Depth Plots}

The output of an Oases run is the transfer function, evaluated on the points of a grid in range and depth. By looking at the Oases transfer functions at a single frequency, the transmission loss at that frequency can be measured in a plot of the complex pressure field as a function of range and depth. Example range-depth plots are shown in Figure 3-5, for $11.5 \mathrm{kHz}$, the center of the band in interest. These plots show the total transmission loss, but do not give information about individual propagation paths. 

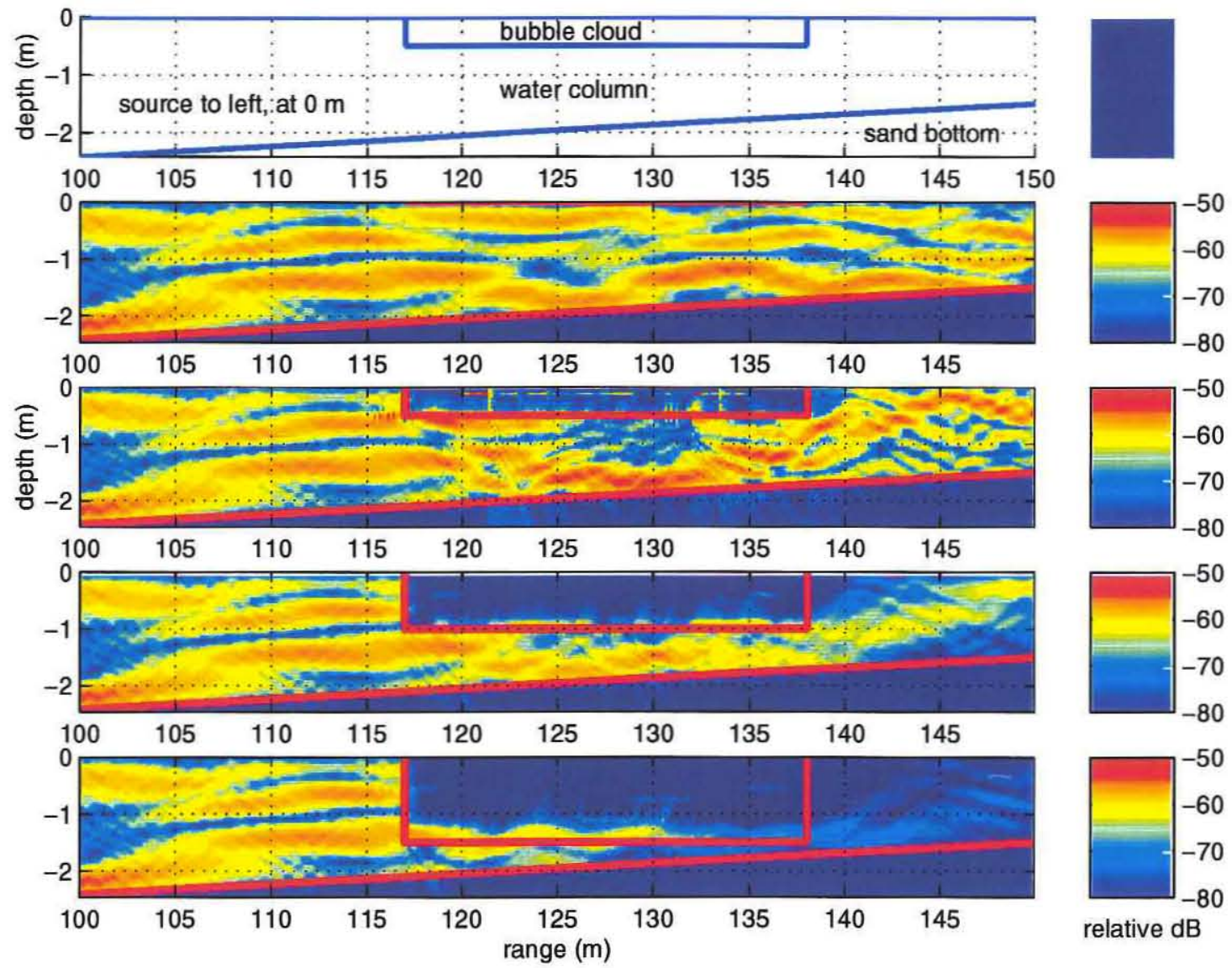

relative $\mathrm{dB}$

Figure 3-5: Transmission loss plots at $11.5 \mathrm{kHz}$. The top plot shows the modeled environment. The plots below are for no bubble cloud, and bubble clouds $0.5 \mathrm{~m}, 1.0 \mathrm{~m}$, and $1.5 \mathrm{~m}$ deep, respectively. The energy scale is in $\mathrm{dB}$ relative to $0 \mathrm{~dB}$ at the source. Red lines indicate the bottom and the bubble cloud boundaries. 
With no bubble cloud, the range-depth plots of the pressure field magnitude show a modal interference pattern characteristic of monochromatic propagation up a wedge, shown in Figure 3-5b. With a $0.5 \mathrm{~m}$ bubble cloud, in Figure 3-5c, the energy upwedge of the beginning of the cloud is compressed vertically in the water column, concentrating in one band with a high pressure magnitude. When the cloud is $1.0 \mathrm{~m}$ deep, there is again a single band of peak pressure magnitudes once the cloud begins, but it is significantly attenuated compared with the case with the $0.5 \mathrm{~m}$ cloud, by approximately $10 \mathrm{~dB}$. For the case with a $1.5 \mathrm{~m}$ deep bubble cloud, the attenuation beyond the cloud is significant, but, as with the other cases, the energy redistributes to again fill the water column.

The bubble clouds were modeled as regions with discrete steps in void fraction, leading to discrete steps in sound speed and attenuation. The modeled clouds had four steps in void fraction. Larger numbers of steps led to numerical convergence problems, some remnant of which can be seen as a high-intensity horizontal line in Figure $3-5 \mathrm{c}$, for the $0.5 \mathrm{~m}$ bubble cloud case.

\section{Impulse Responses}

An additional way to look at the data is to take its Fourier transform, yielding time-domain impulse responses for each point in the range-depth grid. The impulse response's spread in the time domain is generally due to the variety of propagation paths in the environment. If a direct path exists, the direct-path arrival is typically the largest and earliest in the impulse response, followed by increasingly attenuated arrivals from paths with reflections from the surface and bottom.

Transforming the data in this way yields plots which are most conveniently displayed as the impulse responses (pressure field magnitude as a function of time, or delay), stacked vertically as a function of depth. Figure 3-6 shows the impulse responses as a function of depth before the cloud, for varying cloud depths; Figure 3-7 shows them halfway through the cloud; finally, Figure 3-8 show the impulse responses after the bubble cloud.

Before the bubble cloud, there is essentially no difference in the propagation, so all the plots in Figure 3-6 are very similar. In the center of the bubble cloud, Figure 3-7, the impulse responses have lost some energy, and are constrained vertically by the bottom of the cloud and the sandy bottom, but are otherwise similar in character to those before the cloud. After the cloud, the impulse responses contain still less energy, but also the trailing edge of the impulse response is 


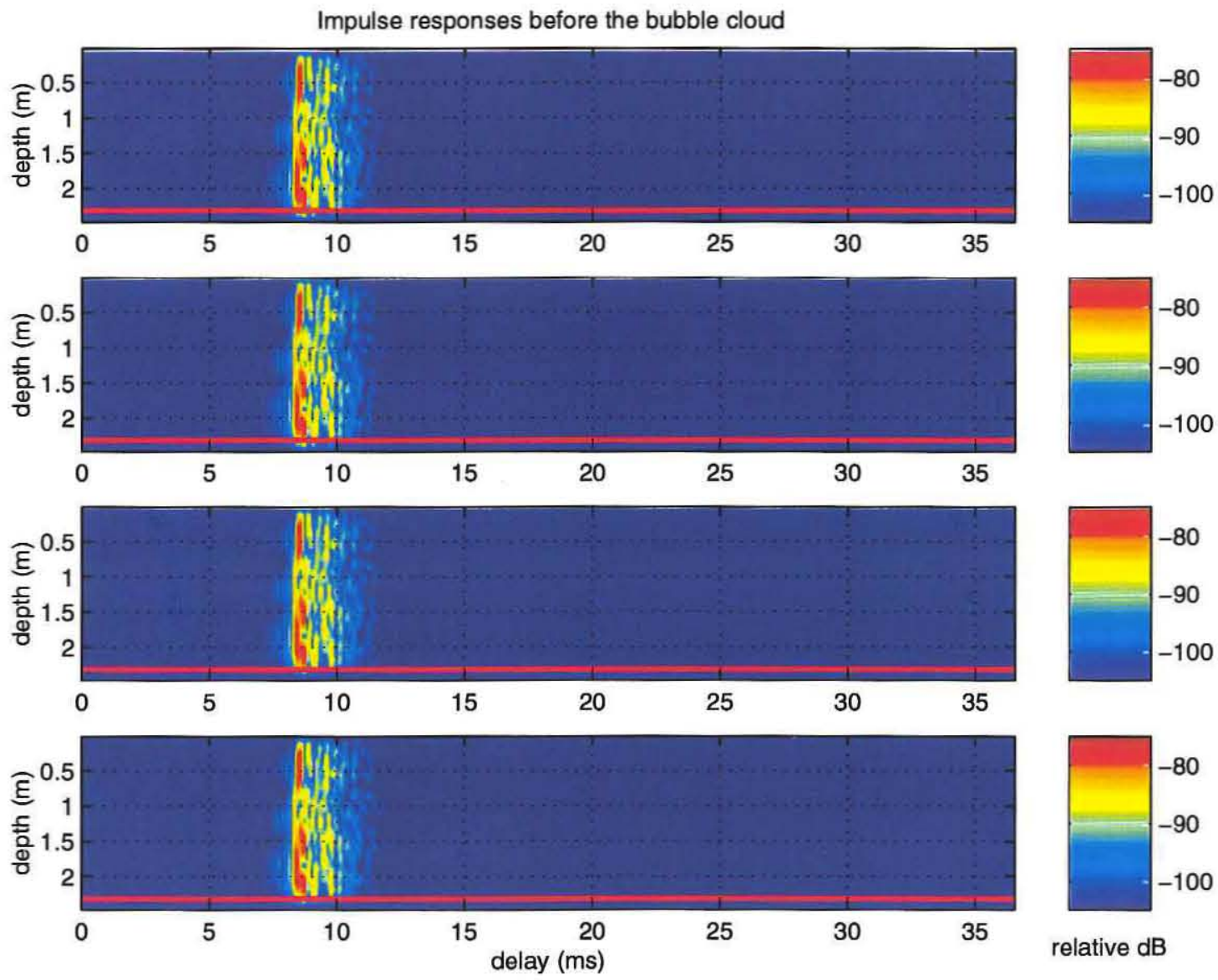

Figure 3-6: Impulse responses, before reaching the bubble cloud, in $2.3 \mathrm{~m}$ of water. The vertical axis is depth, and the horizontal axis is delay. From top to bottom, the plots are for no cloud, a cloud $0.5 \mathrm{~m}$ deep, $1.0 \mathrm{~m}$ deep, and $1.5 \mathrm{~m}$ deep. A red line indicates the bottom depth. 


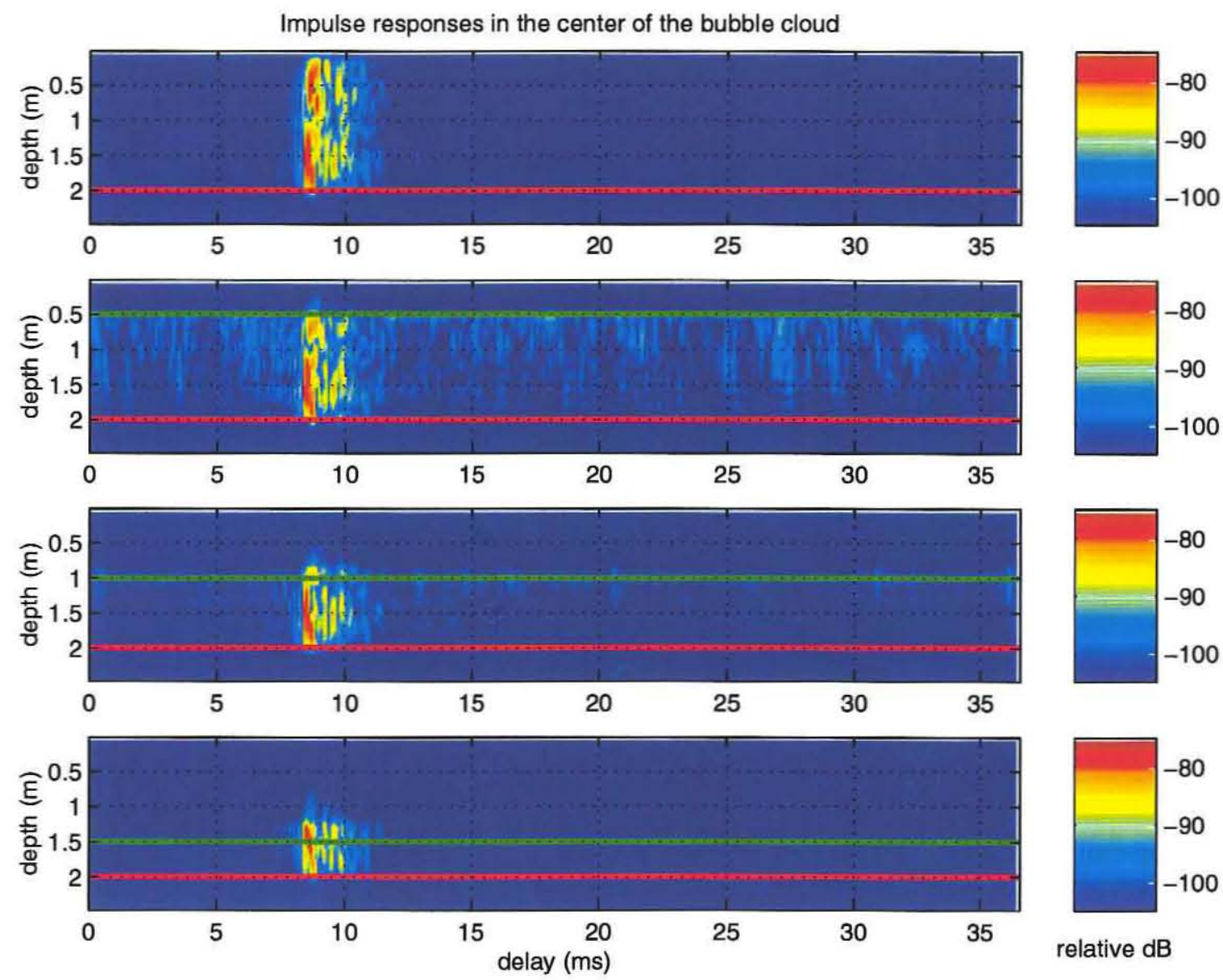

Figure 3-7: Impulse responses, in the center of the bubble cloud, in $2.0 \mathrm{~m}$ of water. The vertical axis is depth, and the horizontal axis is delay. From top to bottom, the plots are for no cloud, a cloud $0.5 \mathrm{~m}$ deep, $1.0 \mathrm{~m}$ deep, and $1.5 \mathrm{~m}$ deep. A red line indicates the bottom depth, and a green line indicates the bottom of the bubble cloud. 

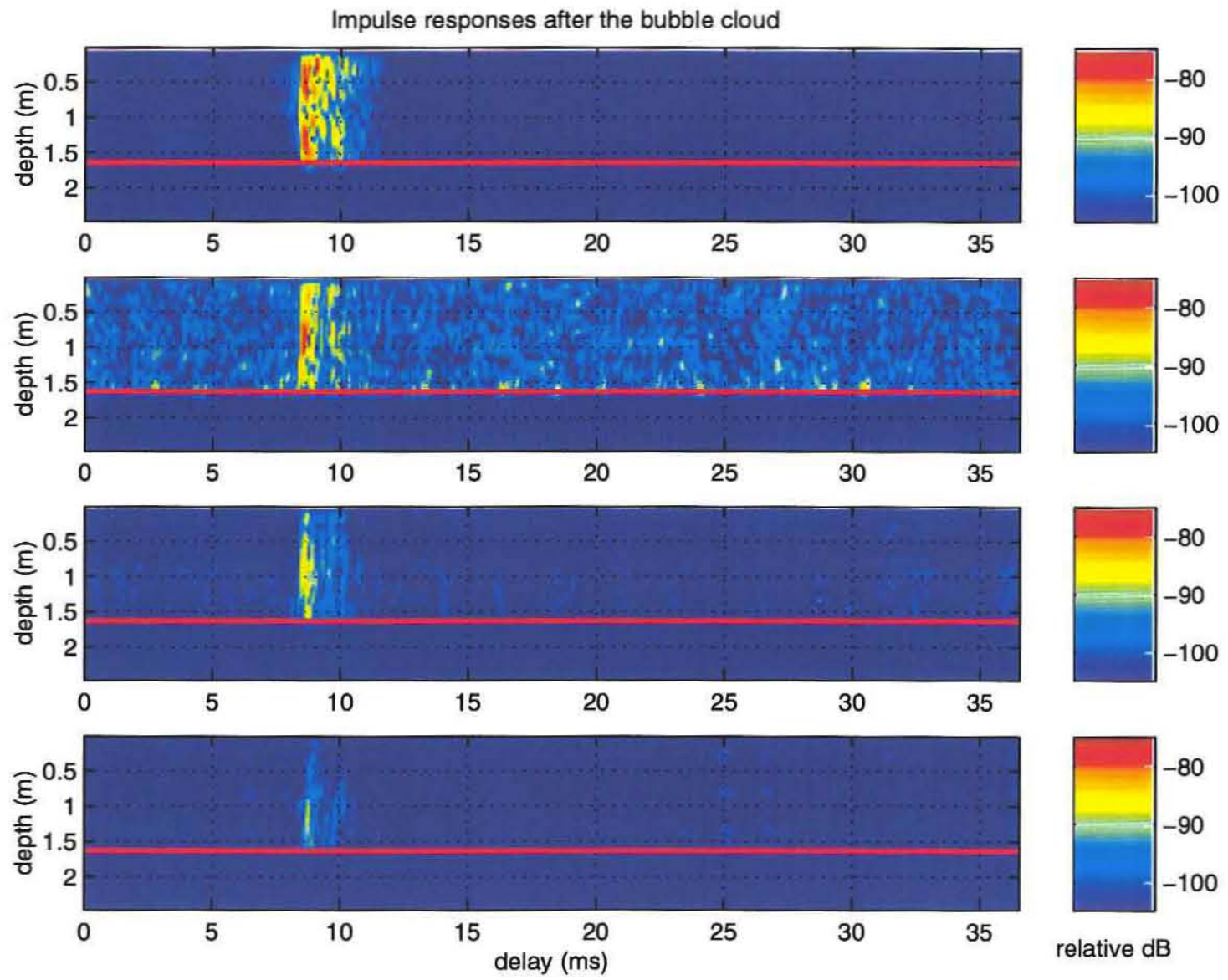

Figure 3-8: Impulse responses, after the bubble cloud, in $1.6 \mathrm{~m}$ of water. The vertical axis is depth, and the horizontal axis is delay. From top to bottom, the plots are for no cloud, a cloud $0.5 \mathrm{~m}$ deep, $1.0 \mathrm{~m}$ deep, and $1.5 \mathrm{~m}$ deep. A red line indicates the bottom depth. 


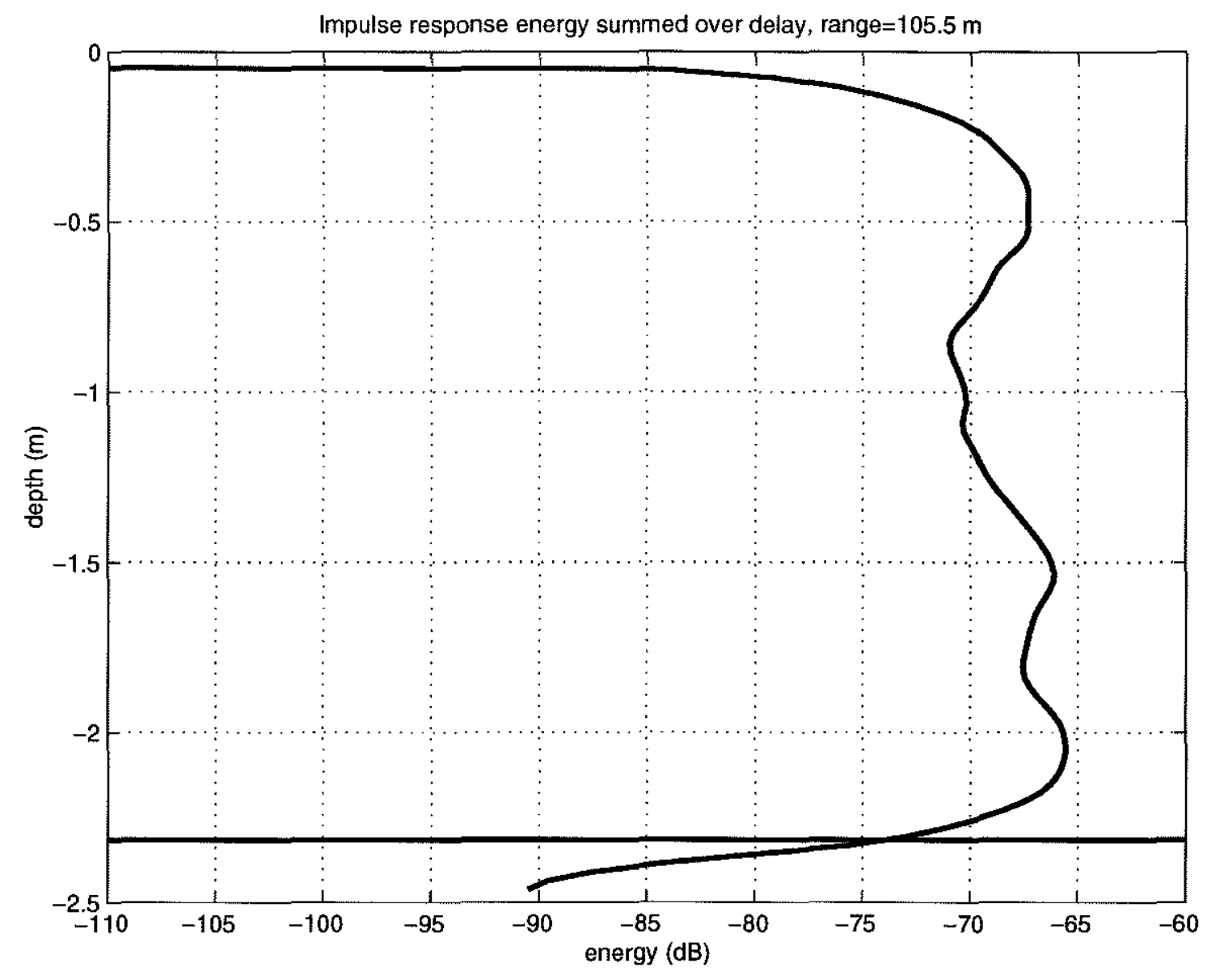

Figure 3-9: Summed energy in the impulse responses as a function of depth, before the bubble cloud.

increasingly attenuated towards the water surface. The leading edge of the impulse response therefore appears to be the first part of the impulse response to regain its energy after the bubble cloud ends. This can be seen in Figure 3-8. The numerical convergence problems with the $0.5 \mathrm{~m}$ bubble cloud depth can be seen in these plots of impulse responses as additional energy across the entire delay axis.

Summing the energy in the impulse responses over the delay axis gives the total energy at a particular depth. Figures 3-9, 3-10, and 3-11 show the summed impulse response energy as a function of depth before, within, and after the bubble cloud, for $0.0 \mathrm{~m}, 0.5 \mathrm{~m}, 1.0 \mathrm{~m}$, and $1.5 \mathrm{~m}$ cloud depths.

Before the cloud, there is essentially no difference due to cloud depth; for all cloud depths, the summed energy is the same, as shown in Figure 3-9. At the range of the center of the cloud, 


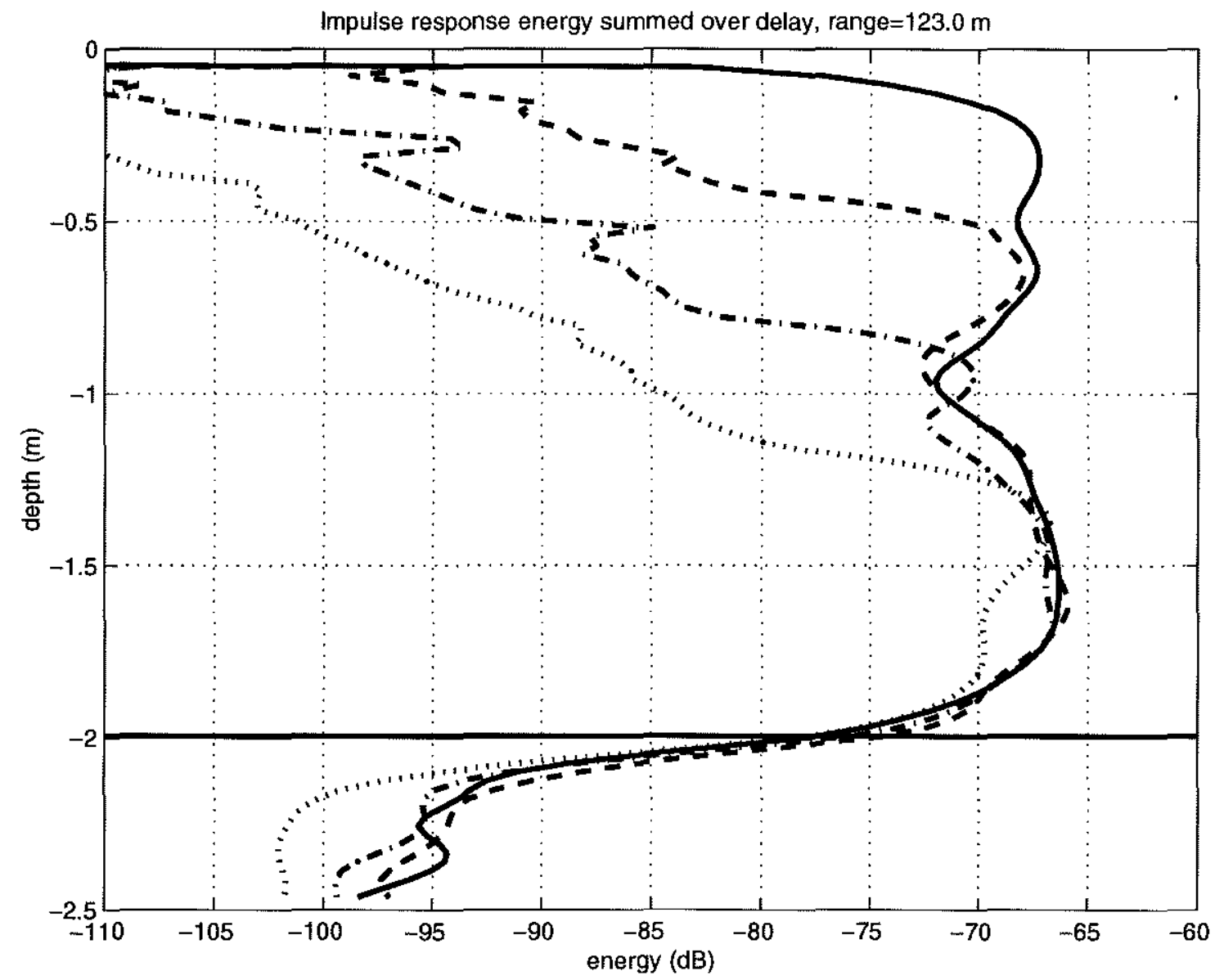

Figure 3-10: Summed energy in the impulse responses as a function of depth, at the range in the center of the bubble cloud. The cases for no cloud, and clouds with depths of $0.5 \mathrm{~m}, 1.0 \mathrm{~m}$, and $1.5 \mathrm{~m}$ are plotted with solid, dashed, dash-dot, and dotted lines, respectively. 


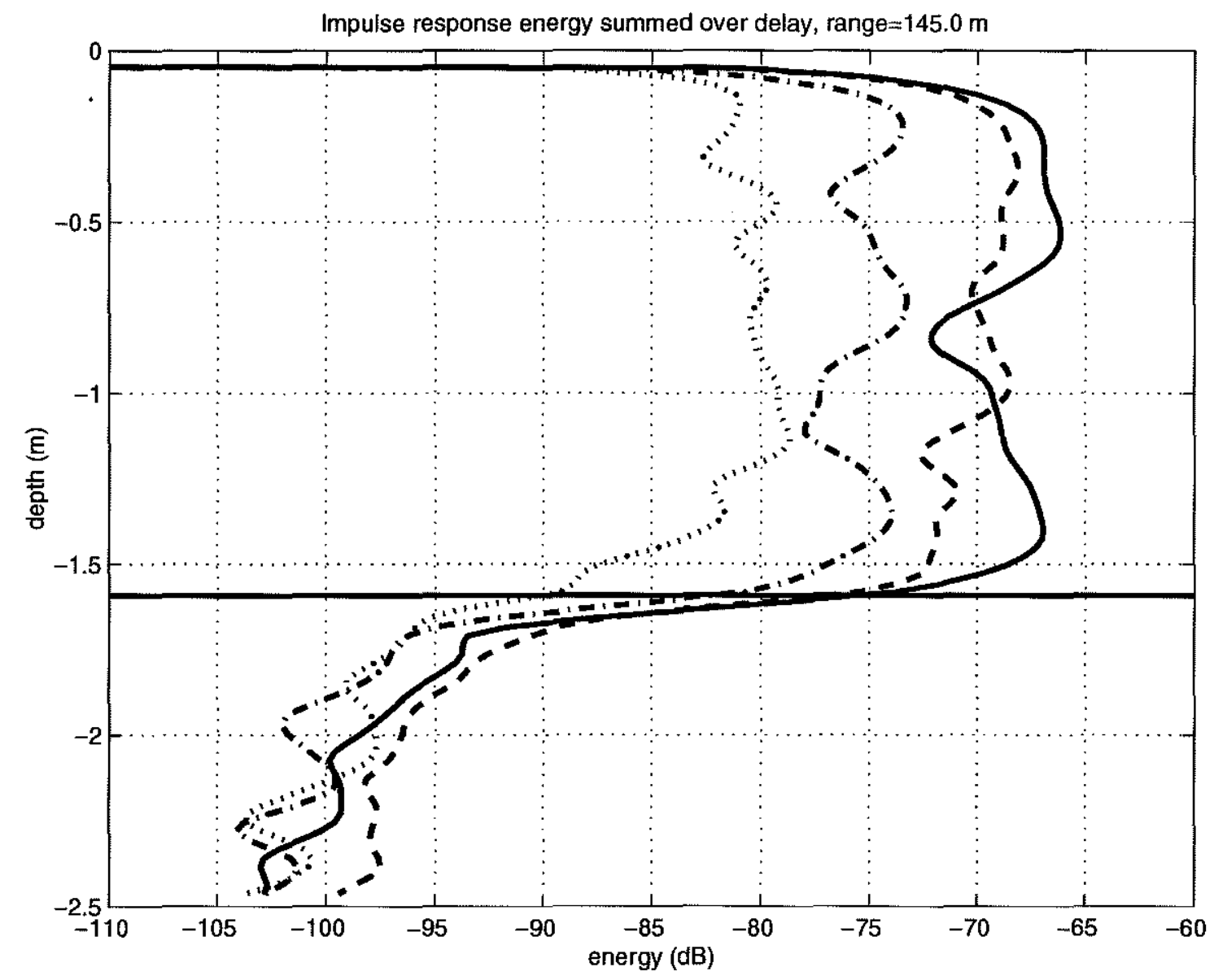

Figure 3-11: Summed energy in the impulse responses as a function of depth, beyond the bubble cloud. The cases for no cloud, and clouds with depths of $0.5 \mathrm{~m}, 1.0 \mathrm{~m}$, and $1.5 \mathrm{~m}$ are plotted with solid, dashed, dash-dot, and dotted lines, respectively. 


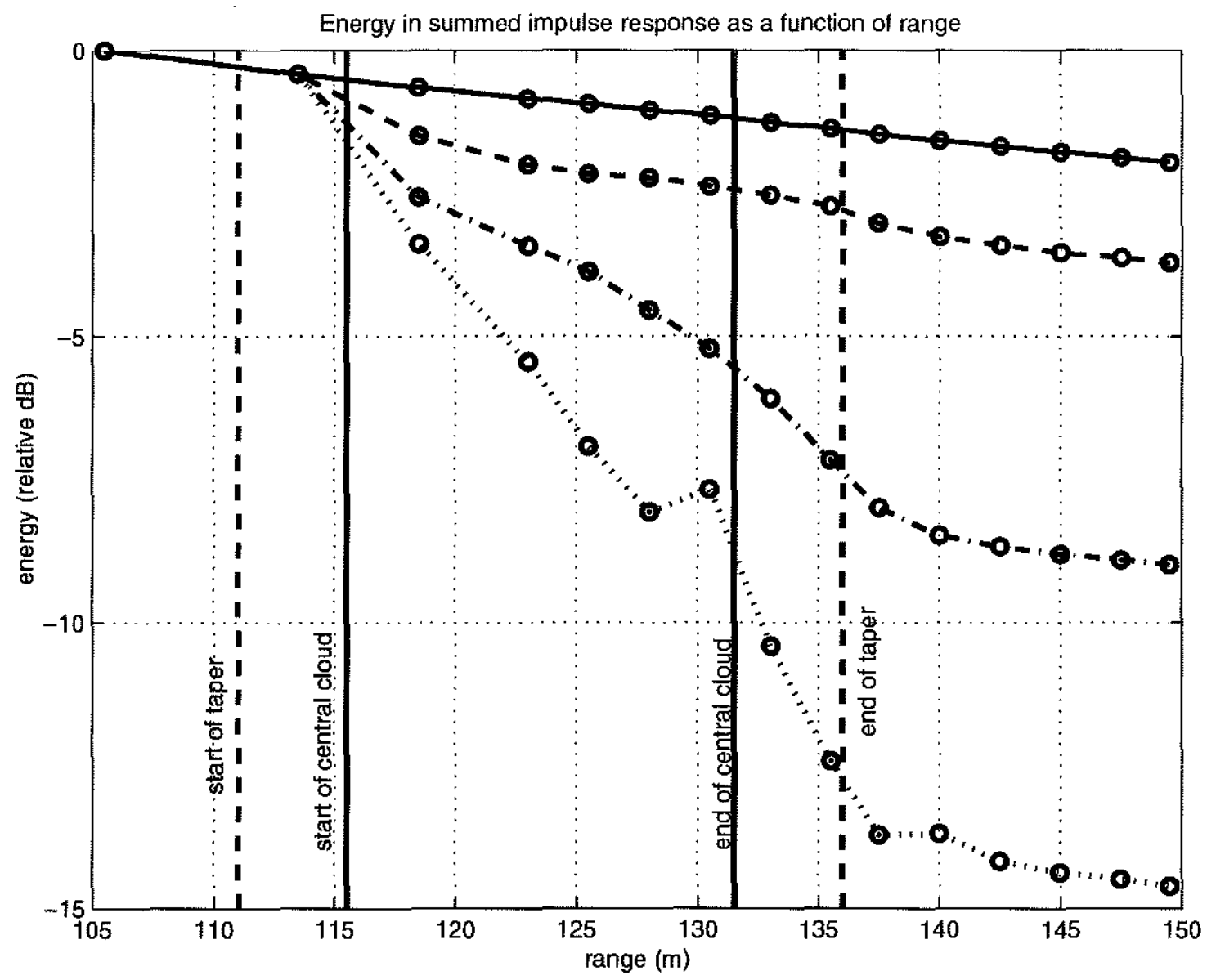

Figure 3-12: Signal energy loss as a function of range and bubble cloud depth. Beyond the bubble cloud, the loss curves in each case are approximately parallel, indicating that the energy redistributes into similar mode structures despite the different bubble cloud depths. The cases for no bubble cloud, and clouds of depth $0.5 \mathrm{~m}, 1.0 \mathrm{~m}$, and $1.5 \mathrm{~m}$ are plotted in solid, dashed, dash-dot, and dotted lines, respectively.

the energy below the cloud is very similar for the different cloud depths, although with increasing cloud depth, the energy propagating through the cloud is increasingly less, shown in Figure 3-10. After the cloud, the energy redistributes throughout the water column; although there is less energy beyond the cloud for the deeper clouds, there is not a significant difference in the vertical distribution of energy for the various clouds.

The region below the bubble cloud can be viewed as a constraining waveguide, since little energy propagates in the high-attenuation cloud. Upon passing the cloud, the energy quickly redistributes into approximately the same modal structure, regardless of the cloud depth. Further propagation beyond the clouds does not depend on the bubble cloud depth. Figure 3-12 shows the energy prop- 


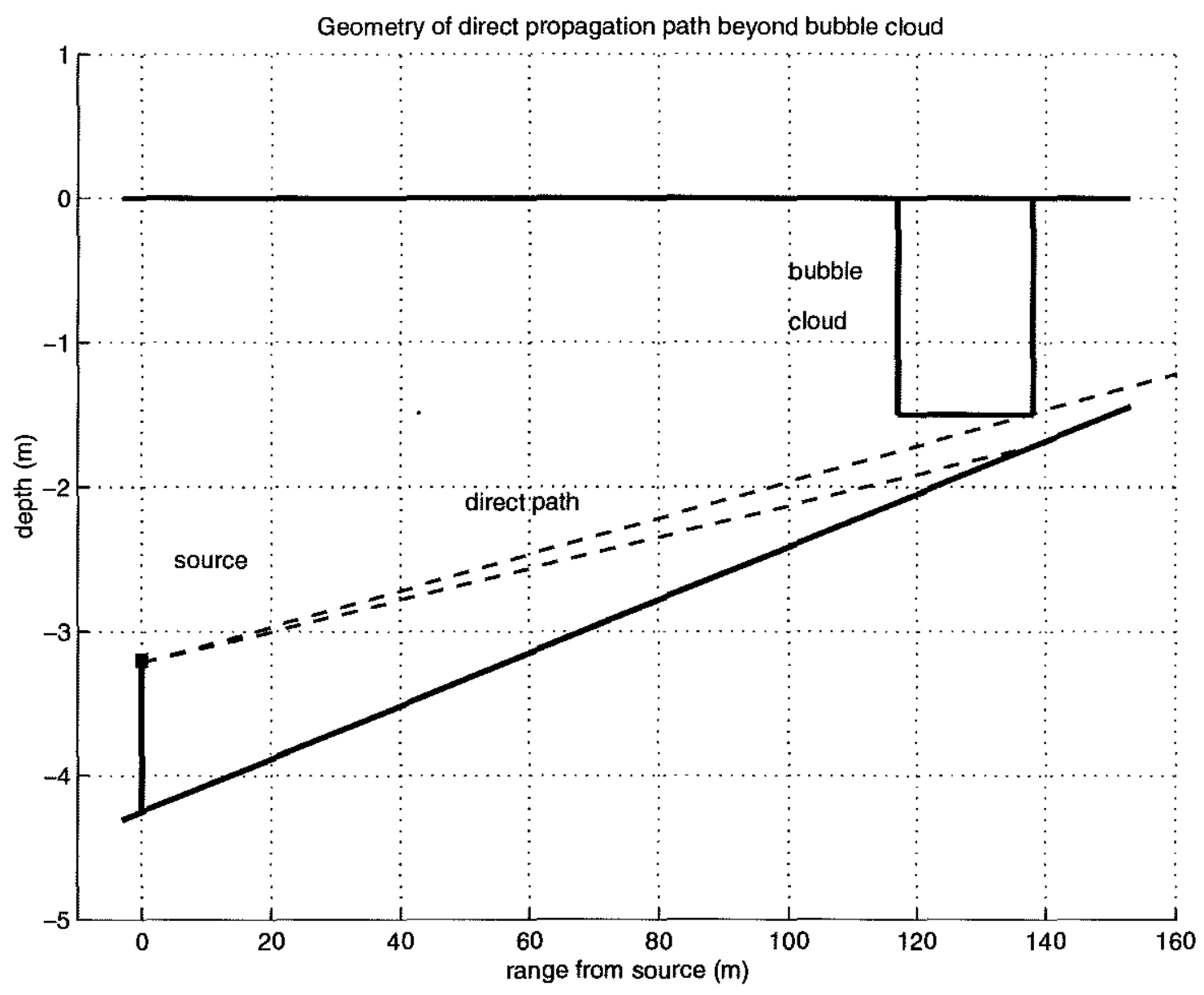

Figure 3-13:

agating beyond the cloud; although the deeper clouds attenuate the energy more heavily, beyond the cloud, the additional energy loss as a function of range is independent of cloud depth.

Even for the deepest bubble cloud in the simulation runs, $1.5 \mathrm{~m}$, some geometric ray paths pass directly under the cloud into the region beyond without reflecting off the bottom. These rays which pass under the cloud correspond to the primary, direct-arrival, peak of the impulse response. If a geometric ray model is sufficient to explain the propagation in the region of the bubble cloud, then the energy in the direct arrival peak of the impulse response should be independent of the cloud depth, as these paths do not intersect even the deepest cloud.

The geometry for the direct paths to regions beyond the bubble cloud is shown in Figure 3-13. At each range beyond the bubble cloud, a small window of depths has a direct path to the source even 

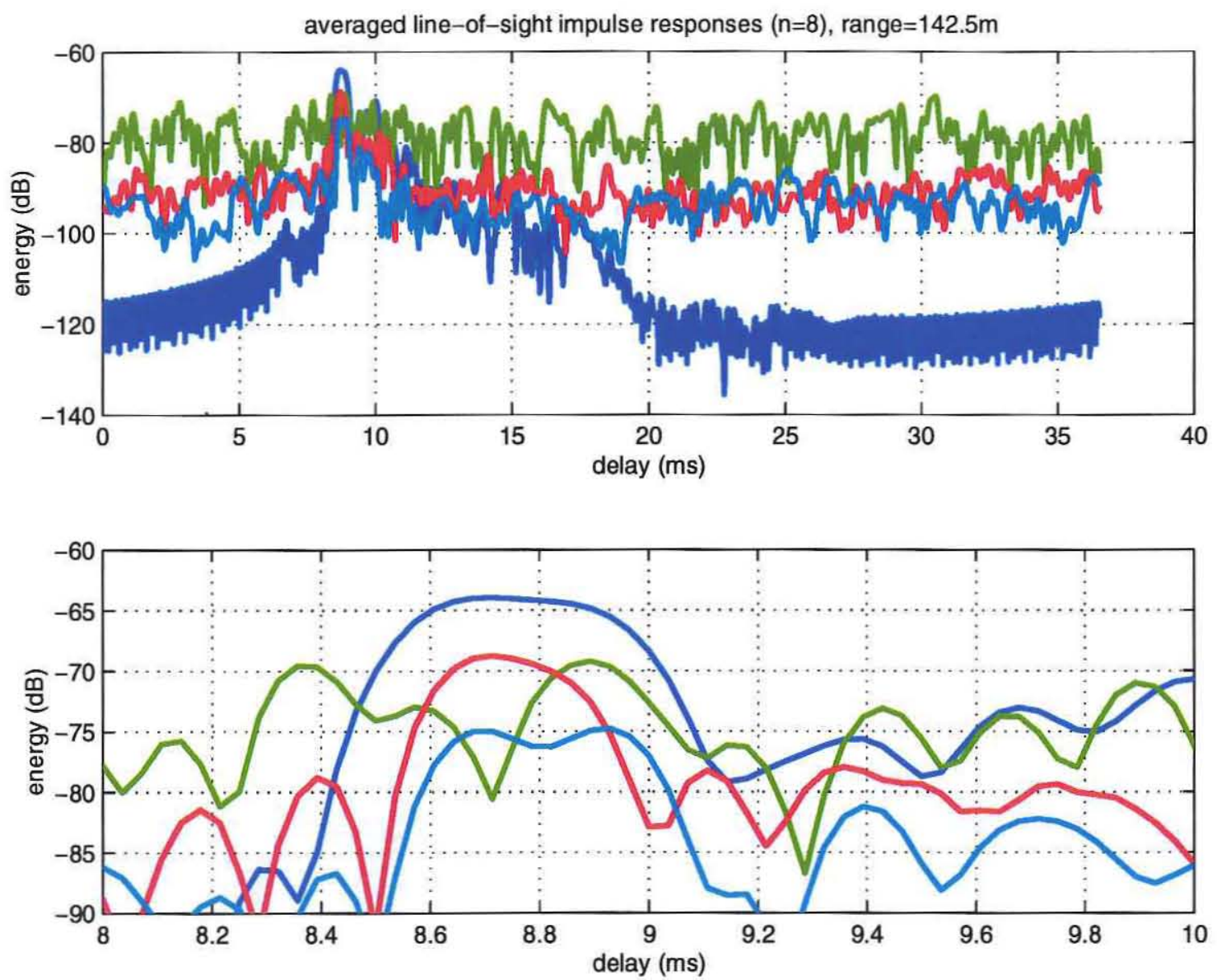

Figure 3-14: Averaged impulse responses for the window of direct paths beyond the bubble cloud. The lower plot shows an expanded view around the main arrival. The cases with no bubble cloud, and with clouds of depth $0.5 \mathrm{~m}, 1.0 \mathrm{~m}$, and $1.5 \mathrm{~m}$ are plotted in dark blue, green, red, and cyan, respectively. Although a geometric ray model would not predict attenuation of the main arrival due to the presence of the bubble cloud, the runs with bubble clouds have an attenuated main arrival, indicating that a geometric ray model may not be sufficient.

with a $1.5 \mathrm{~m}$ cloud, ranging between 7 and 9 depths with the depth sampling of $2.6 \mathrm{~cm}$. With a geometric ray model, the direct-arrival peak of the impulse response, the primary peak, should be unaffected by the presence of a bubble cloud which the rays do not intersect. The average impulse responses over this window of depths for which there are direct arrival are plotted in Figure 3-14. Also shown in that figure is an expanded view of the direct-arrival peak. The direct-arrival peak for the cases with bubble clouds are attenuated compared with the direct-arrival peak with no cloud, indicating that the geometric ray model is most likely not sufficient for modeling shallow water propagation under bubble clouds. The specific attenuations with respect to the case with no bubble cloud are $5.2 \mathrm{~dB}, 4.8 \mathrm{~dB}$, and $10.8 \mathrm{~dB}$, for an $0.5 \mathrm{~m}, 1.0 \mathrm{~m}$, and $1.5 \mathrm{~m}$ bubble cloud, respectively. 


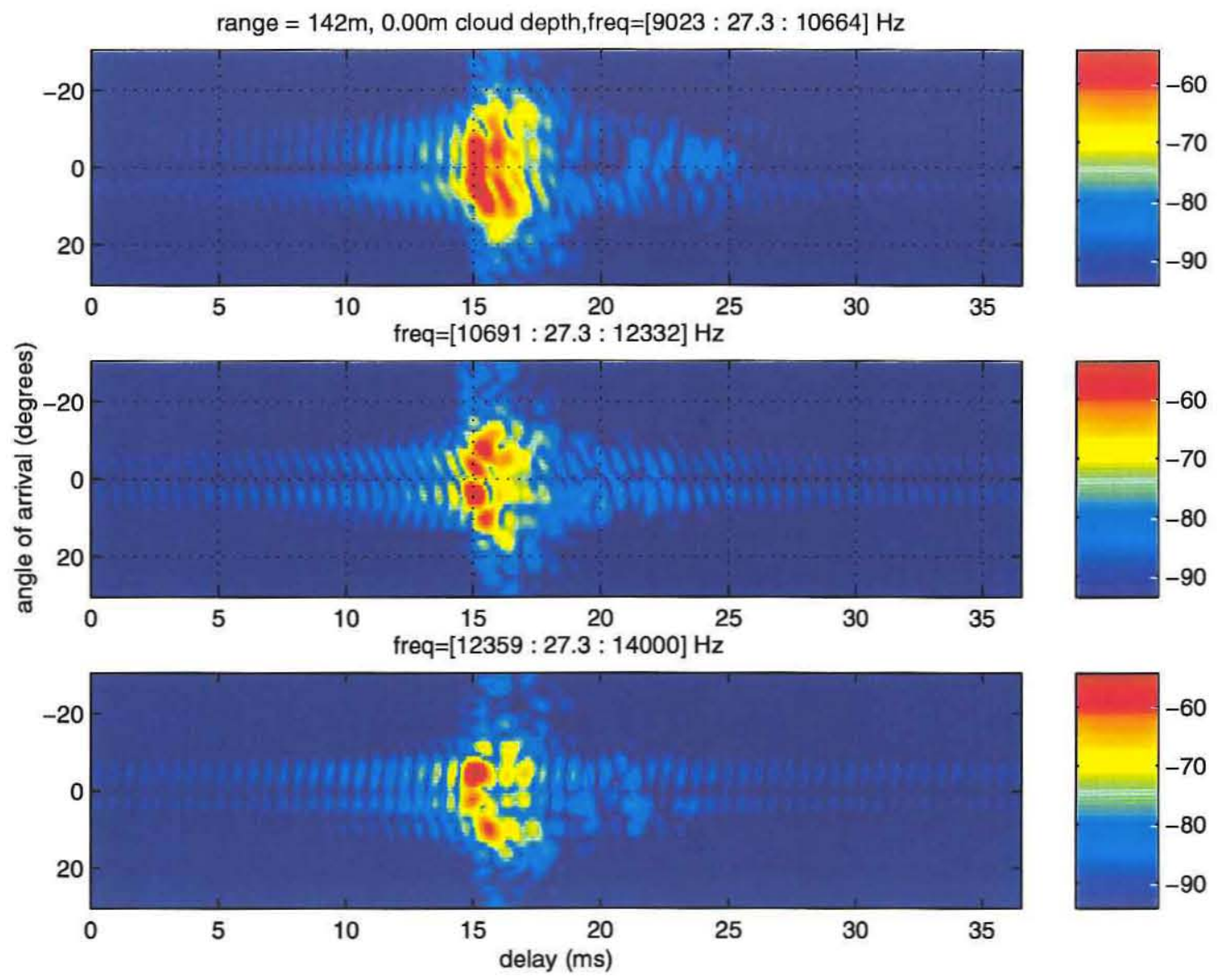

Figure 3-15: Angle of arrival of impulse responses, for no bubble cloud. The three plots cover three equal subbands across the $9-14 \mathrm{kHz}$ band, with the lowest frequency subband in the top plot.

\section{Angle of Arrival}

As a final method of viewing the results of the simulation, the impulse responses as a function of depth can be beamformed to show arrival energy as a function of delay and angle.

Figures 3-15, 3-16, and 3-17 show the angle-of-arrival plots for no bubble cloud, and clouds with depths of $0.5 \mathrm{~m}$ and $1.0 \mathrm{~m}$, respectively. Figure 3-18 shows the angle-of-arrival energies summed over delay, yielding arrival energy as a function of angle. The beamforming was performed using only the depths below the cloud, where a uniform sound speed existed which would support plane waves. Due to the heavy attenuation within the bubble clouds, however, beamforming over the entire water column gives essentially the same results, as the contribution from the waves propa- 


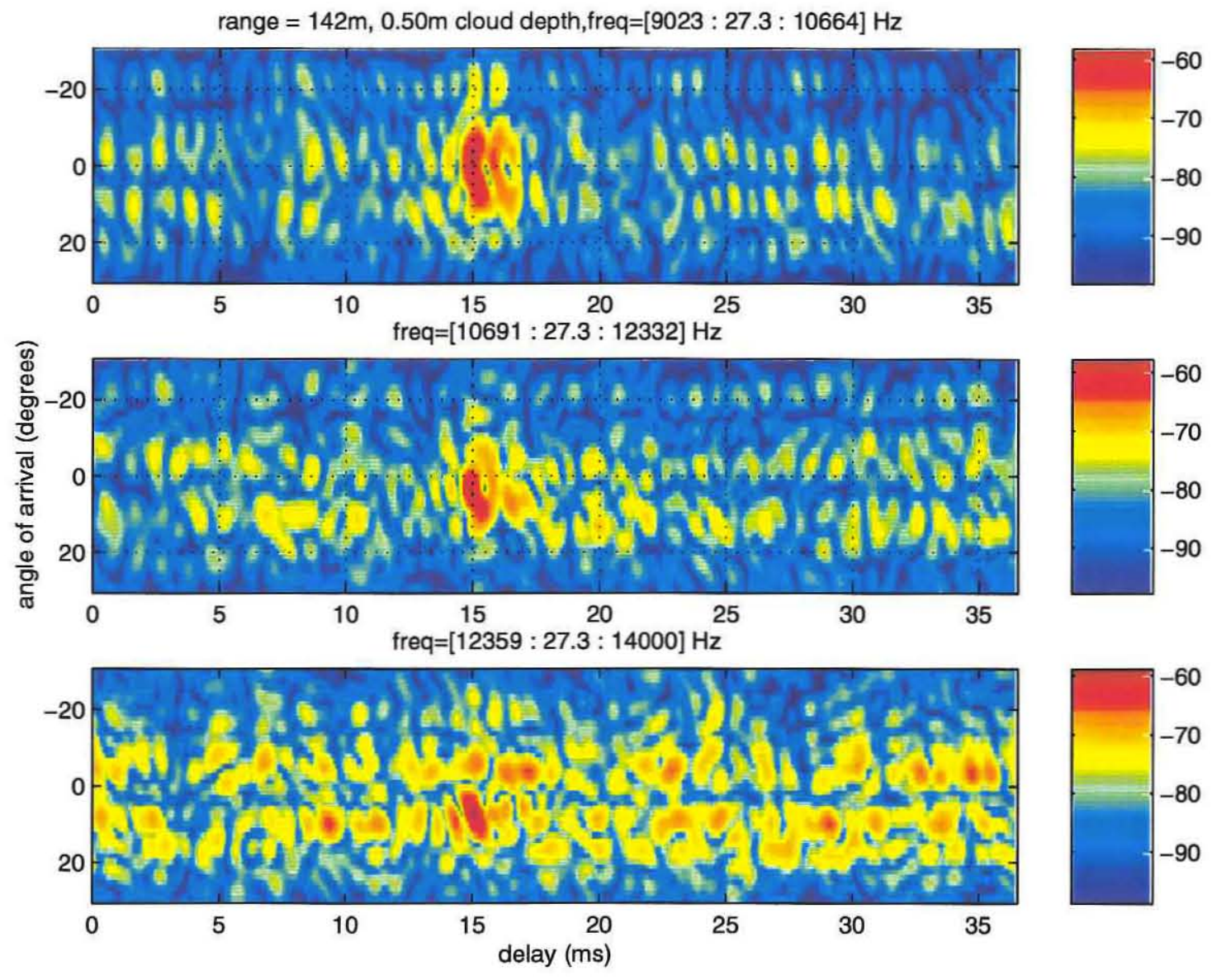

Figure 3-16: Angle of arrival of impulse responses, for a bubble cloud with a depth of $0.5 \mathrm{~m}$. The three plots cover three equal subbands across the $9-14 \mathrm{kHz}$ band, with the lowest frequency subband in the top plot. 


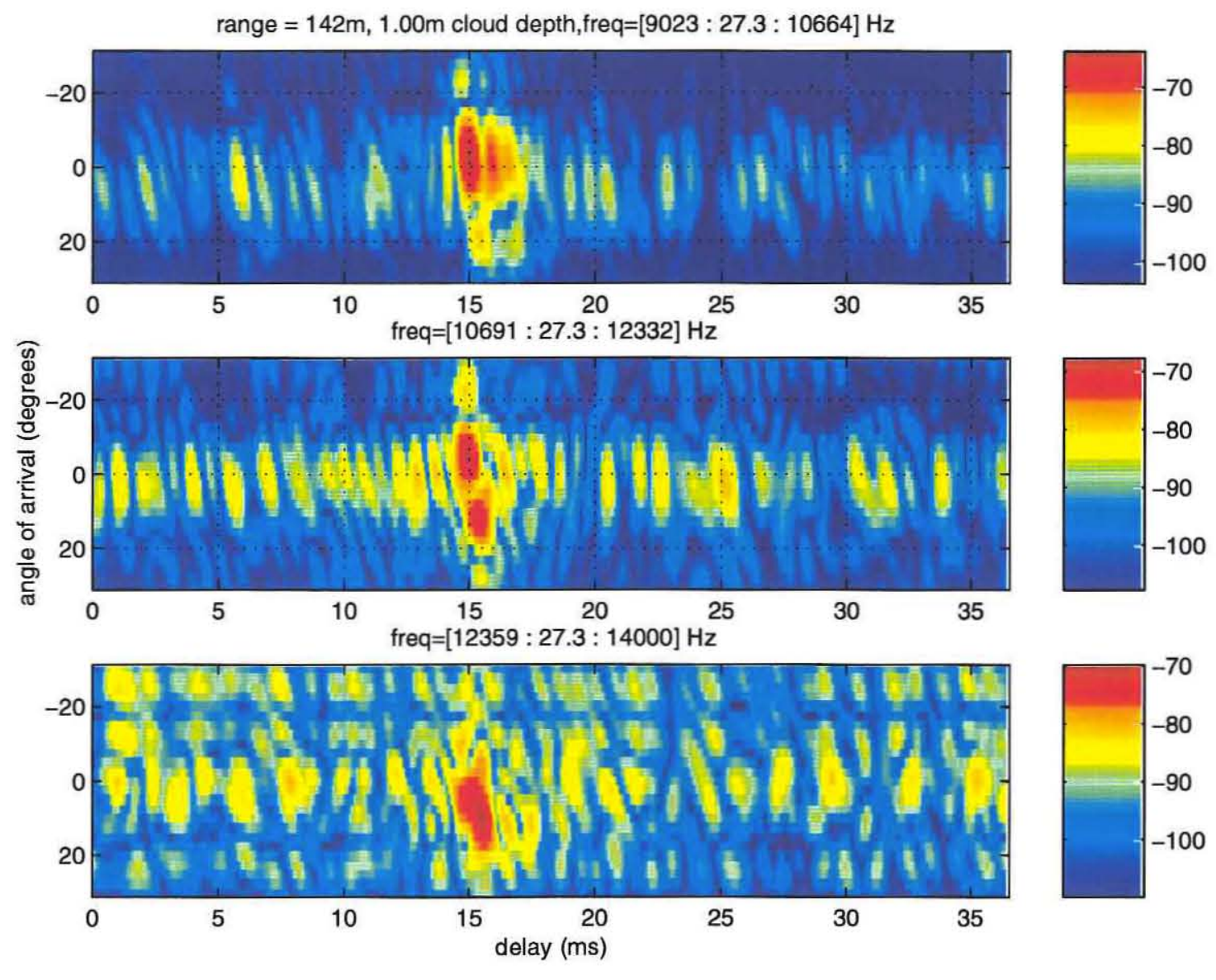

Figure 3-17: Angle of arrival of impulse responses, for a bubble cloud with a depth of $1.0 \mathrm{~m}$. The three plots cover three equal subbands across the $9-14 \mathrm{kHz}$ band, with the lowest frequency subband in the top plot. 

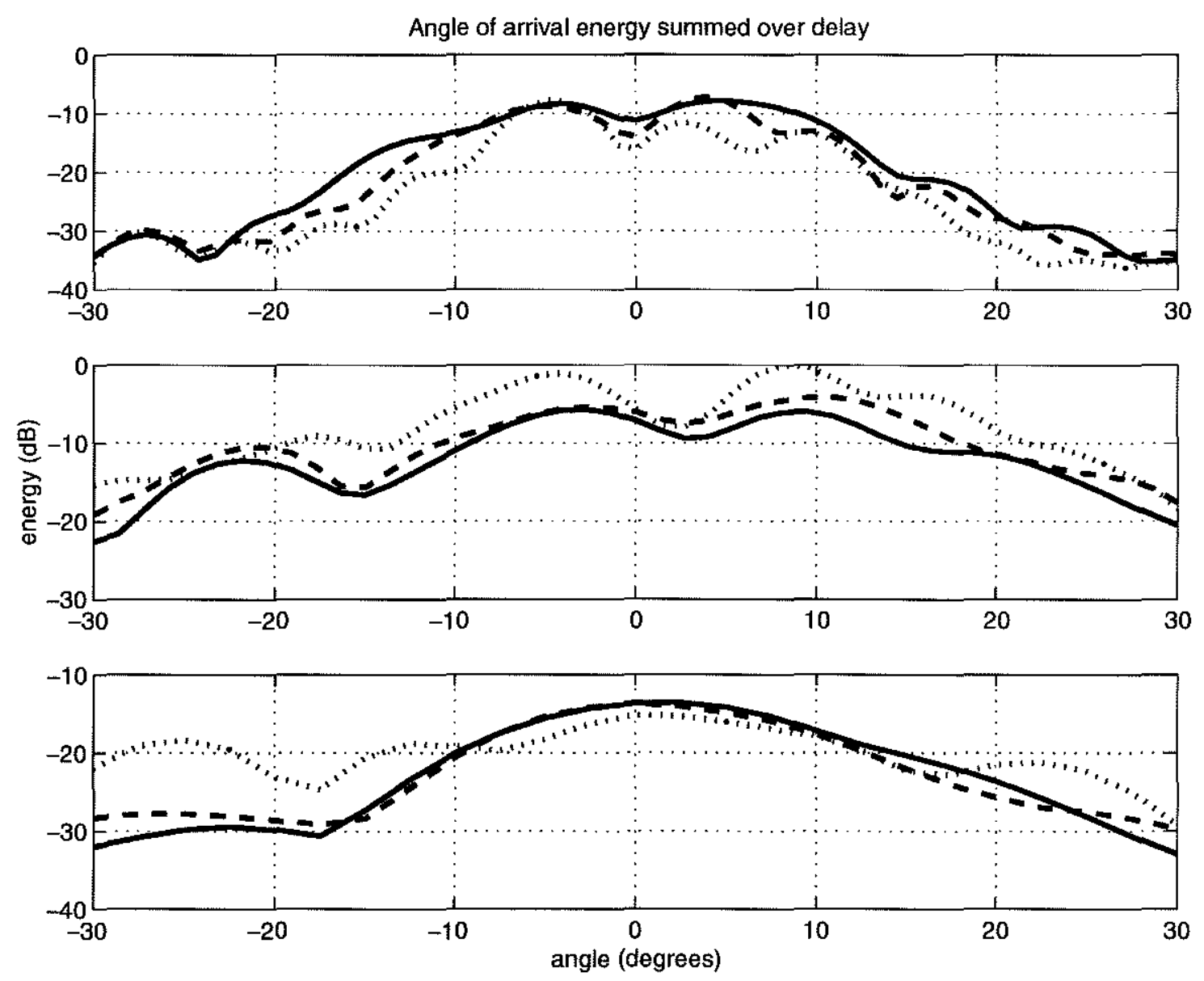

Figure 3-18: Energy in angle-of-arrival plots, summed over delay, yielding total energy received as a function of angle. Results for no bubble cloud, and clouds with depth $0.5 \mathrm{~m}$ and $1.0 \mathrm{~m}$ are plotted with solid, dashed, and dotted lines, respectively. 
gating through the cloud is negligible.

\subsection{Conclusions}

The Oases propagation simulations showed that geometric ray models may not be sufficient for modeling shallow water, surf zone acoustic propagation. The results using a full wave equation integration showed an excess attenuation in direct-path arrival energy due to the presence of bubble clouds, which would not be predicted by geometric ray models. Due to the numerical convergence problems which occurred, in particular at the interfaces between layers in the bubble clouds, these results cannot be considered conclusive. They do, however, provide evidence that more involved propagation models may be required when modeling the acoustic communication channel in the surf zone. 


\section{Bibliography}

[1] A. B. Baggeroer, "Acoustic Telemetry - An Overview," IEEE J. Oceanic Eng., Vol. OE-9, No. 4, pp. 229-235, Oct. 1984.

[2] J. A. Catipovic, "Performance Limitations in Underwater Acoustic Telemetry," IEEE J. Oceanic Eng., Vol. 15, No. 3, pp. 205-216, July 1990.

[3] C. S. Clay, H. Medwin, Acoustical Oceanography. New York: John Wiley \& Sons, 1977.

[4] K. W. Commander, A. Prosperetti, "Linear pressure waves in bubbly liquids: Comparison between theory and experiment," J. Acoust. Soc. Amer., Vol. 85, No. 2, pp. 732-746, Feb. 1989.

[5] G. Deane, D. Stokes, "Near Shore Acoustic Network Experiment: A collaborative experiment, May 6-21, 1999," preliminary report, Scripps Institution of Oceanography, June 1999.

[6] D. M. Farmer, G. B. Deane, S. Vagle, "The Influence of Bubble Clouds on Acoustic Propagation in the Surf Zone," submitted to IEEE J. Oceanic Eng, special issue on high-frequency acoustics, 2000.

[7] M. Feder, A. V. Oppenheim, E. Weinstein, "Maximum Likelihood Noise Cancellation Using the EM Algorithm," IEEE Trans. Acoust., Speech, Signal Processing, Vol. 37, No. 2, Feb. 1989.

[8] L. E. Freitag, T. F. Duda, "Acoustic Channel Identification," final report for Woods Hole Oceanographic Institution Proposal No. 6934.1, Oct. 1992.

[9] G. V. Frisk, Ocean and Seabed Acoustics. Englewood Cliffs, New Jersey: Prentice Hall, 1994.

[10] F. B. Jensen, "On the use of stair steps to approximate bathymetry changes in ocean acoustic models," it J. Acoust. Soc. Amer., Vol. 104, No. 3, pp. 1310-1315, Sept. 1998.

[11] F. B. Jensen, W. A. Kuperman, M. B. Porter, H. Schmidt, Computational Ocean Acoustics. New York: American Institute of Physics, 1994. 